\title{
Hercynite-quartz-bearing granulites from Brejões Dome area, Jequié Block, Bahia, Brazil: Influence of charnockite intrusion on granulite facies metamorphism
}

\author{
Johildo Barbosa $^{\mathrm{a}, *}$, Christian Nicollet ${ }^{\mathrm{b}}$, Carlson Leite ${ }^{\mathrm{c}}$, Jean-Robert Kienast ${ }^{\mathrm{d}}$, \\ Reinhardt A. Fuck ${ }^{\mathrm{e}}$, Eron Pires Macedo ${ }^{\mathrm{a}}$ \\ ${ }^{\text {a } C P G G-C e n t r o ~ d e ~ G e o l o g i a ~ e ~ G e o f i ́ s i c a / C u r s o ~ d e ~ P o ́ s-G r a d u a c ̧ a ̃ o ~ e m ~ G e o l o g i a, ~ U n i v e r s i d a d e ~ F e d e r a l ~ d a ~ B a h i a, ~ R u a ~ C a e t a n o ~ M o u r a ~ 123, ~}$ \\ Federação, 40210-350 Salvador, Bahia, Brazil \\ ${ }^{\mathrm{b}}$ Université Blaise Pascal, Département de Géologie, 5, Rue Kessler, 63038 Clermont-Ferrand, France \\ ${ }^{\mathrm{c}}$ PETROBRAS S.A/UN-BA/ATEX/ LG, Avenida Antônio Carlos Magalhães, 1113, Sala 408, Pituba, Salvador, Bahia, 41856-900, Brazil \\ ${ }^{\mathrm{d}}$ Laboratoire de Pétrologie, Université Pierre et Marie Curie, Paris VI, Place Jussieu, Tour 26, 3eme étage, 75252, Paris, France \\ e Instituto de Geociências, Universidade de Brasília, 70910-900 Brasília, Brazil
}

Received 1 June 2005; accepted 30 March 2006

Available online 30 June 2006

\begin{abstract}
In the present study, we describe and discuss the geology of aluminous-magnesian granulites and associated garnet-bearing charnockitic granulite from the Brejões Dome area, located in the Jequié Block, part of the São Francisco Craton in Bahia, Brazil. Investigation of metamorphic mineral assemblages allows the evaluation of $P-T$ conditions for the formation of these rocks, and therefore to obtain constraints for the better understanding of the geological evolution of the area. We conclude that the rocks from the Brejões Dome area were formed under granulite facies conditions of low to intermediate pressure (5-8 kbar). Temperatures determined in samples of aluminous-magnesian granulites collected away from the dome are in the order of $850{ }^{\circ} \mathrm{C}$, similar to those determined elsewhere in the southern part of the Itabuna-Salvador-Curaçá Orogen. However, samples of the same rock type collected close to the Brejões Dome are hercynite+quartz-bearing and record higher temperatures of about $900-1000{ }^{\circ} \mathrm{C}$. It is suggested that the intrusion of the Brejões charnockite diapir was responsible for a local increase in temperature above the peak temperature of regional granulite metamorphism.
\end{abstract}

(C) 2006 Elsevier B.V. All rights reserved.

Keywords: Granulite; Jequié Block; Paleoproterozoic; São Francisco Craton; Spinel+quartz; Ultrahigh-temperature

\footnotetext{
* Corresponding author.

E-mail addresses: johildo@cpgg.ufba.br (J. Barbosa), nicollet@opgc.univ.bpclermont.fr (C. Nicollet), cmml@petrobras.com.br, cmml@ufba.br(C.Leite),jrk@ccr.jussieu.fr (J.-R. Kienast), reinhardt@unb.br (R.A. Fuck), eron@cpgg.ufba.br (E.P. Macedo).
}

\section{Introduction}

Aluminous-magnesian granulites contain a wide variety of mineral assemblages that are sensitive to $P-T$ conditions of metamorphism, and commonly preserve microstrucutures which are helpful to determine the sequence of mineral reactions (Hensen, 1987; Waters, 1991). 

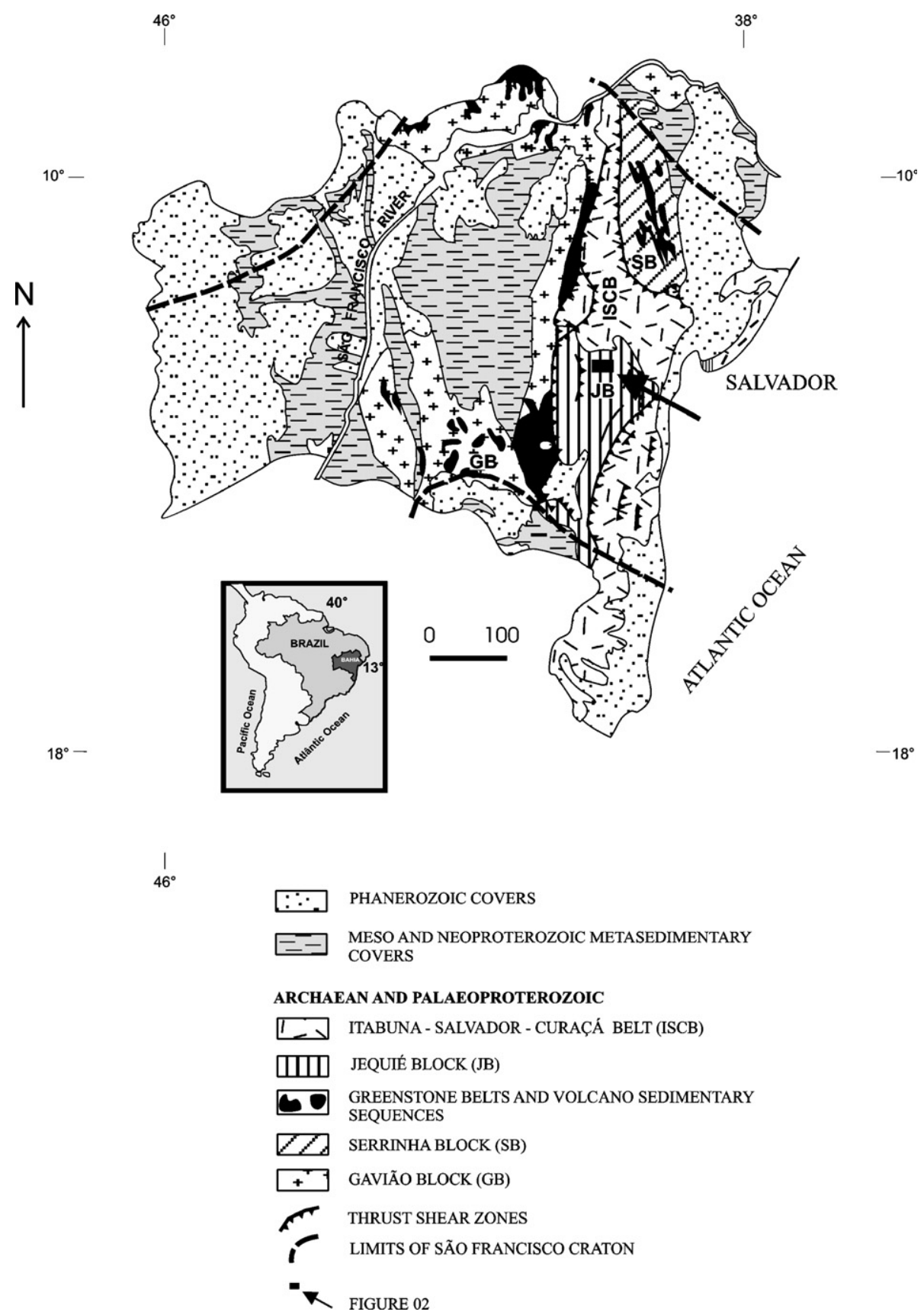

Fig. 1. Geotectonic domains in Bahia (simplified from Barbosa and Dominguez, 1996) with location of the study area.

Quartz-spinel and quartz-hercynite (Zn-poor spinel) assemblages are observed in aluminous-magnesian granulites from a number of areas on Earth. Well known examples are exposed in Antarctica (e.g. Ellis et al., 1980; Sandiford and Powell, 1986; Clarke et al., 1989), Norway (e.g. Tobi et al., 1985), India (e.g. Lal et al., 1987), Australia (e.g. Warren and Hensen, 1989), South Africa (e.g. Waters, 1989), Madagascar (e.g. Nicollet, 1990),
Algeria (e.g. Ouzegane and Boumaza, 1996), and Brazil (Moraes and Fuck, 2000; Moraes et al., 2002). Investigation of these areas and experimental data have shown that the hercynite + quartz association is formed at high temperature and low/intermediate pressure (Waters, 1991). Additionally, the Zn-poor hercynite+ quartz paragenesis is of great interest since it reflects abnormal thermal conditions and an abnormal thermal gradient 


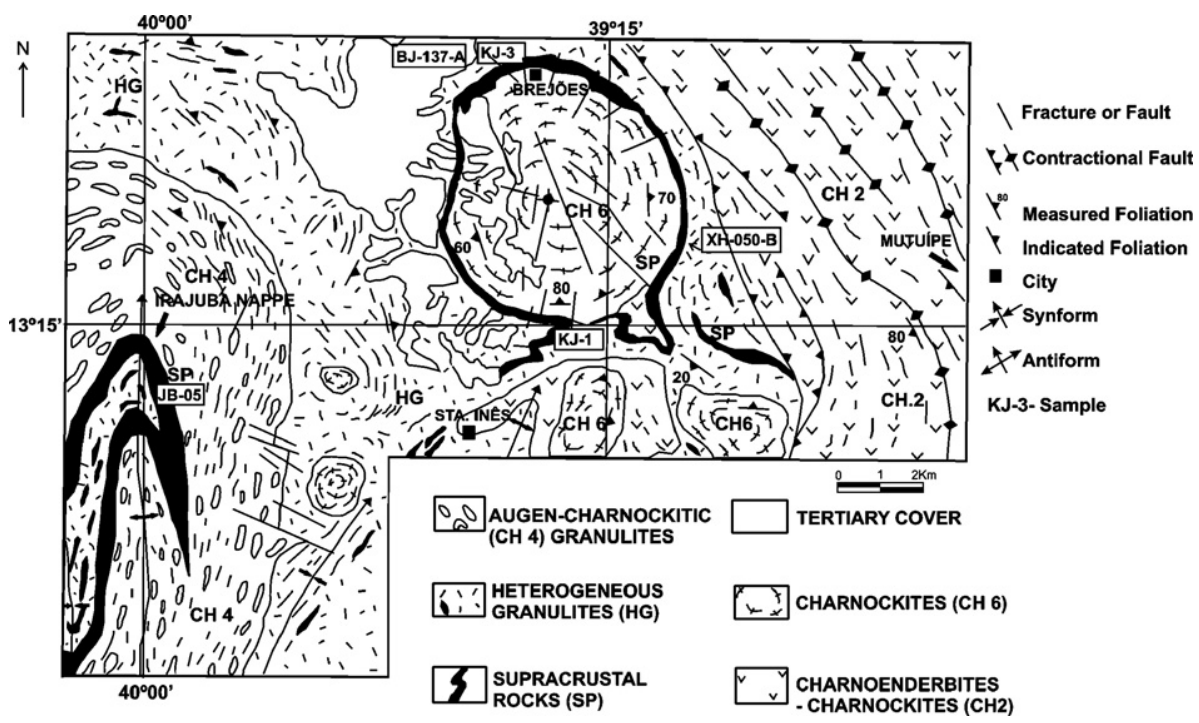

Fig. 2. Simplified geological map of the Brejões Dome area, Jequié Block, Bahia, Brazil (Barbosa et al., 2004).

within the continental crust. Therefore, the study of these granulites may lead to constrain the $P-T$ conditions and the geodynamic context in which they were formed.

In the present study, we describe and discuss the geology of hercynite-quartz-bearing aluminous-magnesian granulites and associated garnet-charnockitic granulites from the Brejões Dome area, in the Jequié Block, Bahia, eastern Brazil. Investigation of metamorphic mineral assemblages has allowed the evaluation of the $P-T$ conditions of formation of these rocks which enhances the understanding of the geologic evolution of this area.

\section{Geological setting}

The study area is located in the northern part of the Jequié Block, Bahia, eastern Brazil. Together with the archaean Gavião and Serrinha blocks, the Jequié Block is part of the basement framework of the São Francisco Craton (Almeida et al., 1976; Almeida, 1977) (Fig. 1). The archaean blocks were amalgamated at ca. $2.08 \mathrm{Ga}$ during a paleoproterozoic collision (Barbosa and Sabaté, 2002, 2004), which gave birth to the highgrade Itabuna-Salvador-Curaçá belt.

The Jequié Block includes enderbitic-charnockitic granulites (CH2), some of which were dated at 2.7-2.8 Ga (Alibert and Barbosa, 1992). They are younger than a very heterogeneous association of orthoderived rocks including charnockitic-tonalitic granulites, mafic and felsic intercalations of varied composition which have $\mathrm{T}_{\mathrm{DM}} \mathrm{Nd}$ model ages between 2.90 and 3.25 Ga (Barbosa et al., 2004). This association is referred to as heterogeneous granulites (HG) in this paper. The HG are associated with granulite facies supracrustal rocks (SP), comprising basaltic metavolcanic rocks, and several types of metasedimentary rocks, e.g. quartzite, iron formation, graphite-rich layers, and aluminous-magnesian granulites which locally contain the assemblage hercynite + quartz, object of this study (Fig. 2). All these rocks display partial melting features, such as migmatitic veins, and occasionally host garnet and cordierite-bearing anatectic granites.

The rocks of the Jequié Block were deformed by three continuous ductile deformation phases, contemporaneous with the paleoproterozoic high-grade metamorphism which affected the region at c.a. 2.1-2.0 Ga (Wilson, 1987; Ledru et al., 1994). The first phase (F1) is represented by large west-verging recumbent folds, with subhorizontal axial planes and $\mathrm{N}-\mathrm{S}$ fold axes, the most spectacular example of which is the Irajuba nappe (Fig. 2). The coaxial second phase (F2) comprises open folds in the Jequié Block and tight folds in the Itabuna-SalvadorCuraçá Belt, both with near vertical axial planes. The third phase (F3), well displayed within the Itabuna-SalvadorCuraçá Belt, is represented by vertical shear zones, which are sub-parallel to the upright axial planes of the tight F2 folds. The last phase gave origin to a vertical foliation with subhorizontal lineations. Kinematic indicators show that movement was top to northeast. Due to the presence of retrograde minerals such as hornblende and biotite formed after orthopyroxene in some transposition corridors, it is suggested that some parts of the high-grade region were reactivated during uplift of the granulite terrain (Barbosa, 1990).

Geothermobarometric data on the high-grade metamorphism in southern Bahia were obtained through 
petrological investigation of rare garnet-bearing mafic granulites. Pressures of about 5-7 kbar and high temperatures between 850 and $870{ }^{\circ} \mathrm{C}$ were determined (Barbosa, 1986, 1990; Barbosa and Fonteilles, 1991).

In this work, previously obtained thermobarometric data will be compared with the results obtained for aluminous-magnesian granulite of the supracrustal rock layers (SP) and garnet-bearing charnockitic granulite from the HG which occur in the Brejões Dome area, in the northern part of the Jequié Block.

\section{Geology of the Brejões Dome area}

The HG (Fig. 2) are exposed in the central part of the study area, surrounding the Brejões, Santa Inês, and several other charnockite domes. This unit, with $\mathrm{T}_{\mathrm{DM}}$ model ages of 2.9-3.2 Ga is essentially formed of an association of charnockitic-tonalitic granulites, with mafic and felsic intercalations of varied composition, equilibrated under granulite facies conditions (Barbosa et al., 2004). HG are usually greenish-gray, and are coarse- to medium-grained. They may be migmatitic, containing orthopyroxene-bearing leucosomes. Some of these rocks have garnet-orthopyroxene-plagioclase-quartz assemblages (Fig. 2). The garnet-bearing charnockitic granulite (sample XH-050-B), collected east of the Brejões Dome, is a dark-green banded rock, with centimetre-wide darker orthopyroxene-rich bands and lighter quartz-mesoperthitedominated bands. Garnet crystals are dispersed within the rock, but tend to be more abundant in the dark bands.

The SP (Fig. 2) occur as enclaves or intercalations within HG. They constitute $20-50 \mathrm{~cm}$ to $1-5 \mathrm{~m}$ thick layers, concordant with the regional rock foliation (Barbosa, 1986, 1990). They sometimes form much larger layers, tens or hundreds of metres thick, as for example that which surrounds the Brejões Dome and in the Irajuba nappe (Fig. 2). The supracrustal rocks comprise mainly mafic granulites (formerly basalts and andesitic basalts), quartzite, iron formation, quartzfeldspar layers, aluminous-magnesian granulites that include hercynite-quartz-bearing granulite, and scattered graphite-rich layers. The aluminous-magnesian granulites are probably the result of metamorphism of pelitic rocks (Barbosa, 1990). In general, these rocks are medium to coarse-grained. The main outcrops are found within the valleys around the Brejões and Santa Inês domes (samples $\mathrm{KJ}-1, \mathrm{KJ}-3, \mathrm{BJ}-137-\mathrm{A}$ ) and in the Irajuba nappe (sample JB-05) in the western part of the study area (Fig. 2). The aluminous-magnesian granulites occur as metre to centimetre thick greenish-red layers, rich in pink garnet crystals, as well as cordierite+spinel clots, within a quartz-feldspar matrix. In some outcrops the aluminous- magnesian granulites are intruded by undeformed garnetand cordierite-bearing granites. Petrological and geochemical data indicate that these granites are S-type intrusions (Barbosa et al., 2004), and they are probably products of partial melting which occurred during peakmetamorphic conditions. They have been dated at around 2.0 $\mathrm{Ga}$ by the $\mathrm{Pb}-\mathrm{Pb}$ evaporation method of single zircon crystal, and by the $\mathrm{Th}-\mathrm{U}-\mathrm{Pb}$ in-situ electron microprobe method on monazite (Barbosa et al., 2004).

The $\mathrm{CH} 2$ (charnoenderbitic-charnockitic granulites) (Fig. 2) exposed around Mutuipe, in the eastern part of the study area, tectonically overly $\mathrm{HG}$ and SP (Fig. 2). U-Pb zircon age determinations indicate that their protoliths are ca. 2.7-2.8 Ga old (Alibert and Barbosa, 1992). They are composed of mesoperthite (30-40\%), quartz $(30 \%)$, antiperthitic plagioclase (10-20\%), hornblende (10$20 \%)$, orthopyroxene-clinopyroxene $(2-5 \%)$; biotite, opaques, zircon and apatite are accessory minerals.

The CH4 (charnockitic augen-type granulites) (Fig. 2), also called the Irajuba type, occurs in the western part of the study area. In its central part, this coarse-grained intrusive body includes a large refolded belt of heterogeneous granulite surrounded by a thick layer of granulitic supracrustal (SP) rocks. The Irajuba charnockitic granulite is essentially composed of large, up to $4 \mathrm{~cm}$ mesoperthite porphyroclasts $(40-50 \%)$, quartz (30$40 \%$ ), antiperthitic plagioclase (5-10\%); hornblende, orthopyroxene, and opaques are accessory minerals.

The CH6 (intrusive charnockite bodies) (Fig. 2) constitute the cores of the Brejões, Santa Inês and several other smaller, unnamed oval-shaped domes. They were dated at ca. 2.0 Ga (Barbosa et al., 2004). CH6 charnockite is formed of mesoperthite (30-40\%), antiperthitic plagioclase $(30 \%)$, quartz $(20 \%)$, hornblende $(10 \%)$, orthopyroxene $(5 \%)$, and clinopyroxene $(2-5 \%)$; opaque minerals (ilmenite, magnetite, pyrite), apatite, and zircon are accessory minerals.

Mineralogy of these rocks is fairly similar, however petrologic and geochemical studies led to identification of different types of parent magmas for these units. Granulite rocks $\mathrm{CH} 2$ and $\mathrm{CH} 4$ are mainly granodioritic to granitic, medium-K calc-alkaline suites. CH6 charnockites of Brejões and Santa Inês domes are more siliceous and are typically granite. Geochemical modelling shows that the syn-dome CH6 charnockites probably are products of melting of a CH2-type precursor (Barbosa et al., 2004).

\section{Petrography}

Samples analysed to constrain high-grade $P-T$ conditions recorded in the study area were collected from aluminous-magnesian granulites, exposed both away 
Table 1

Chemical data and mineral assemblages

\begin{tabular}{|c|c|c|c|c|c|c|c|c|c|c|c|c|}
\hline \multirow[t]{3}{*}{ Samples } & \multicolumn{12}{|c|}{ Peak metamorphic phases } \\
\hline & \multirow[t]{2}{*}{ Grt1 } & Grt1 & \multirow[t]{2}{*}{ Opx1 } & \multirow[t]{2}{*}{$\mathrm{Opx} 1 \mathrm{Al}_{2} \mathrm{O}_{3}$} & \multirow[t]{2}{*}{ Sil1 } & \multirow[t]{2}{*}{ Crd1 } & \multirow[t]{2}{*}{ Spl1 } & \multirow[t]{2}{*}{ Bt1 } & \multirow[t]{2}{*}{ Bt1Ti } & \multirow[t]{2}{*}{ Bt1F } & \multirow[t]{2}{*}{ Pl1 } & \multirow[t]{2}{*}{ Mp1 } \\
\hline & & $\mathrm{Al}_{2} \mathrm{O}_{3}$ & & & & & & & & & & \\
\hline $\mathrm{KJ}-3$ & 0.37 & 21.19 & & & $\mathrm{X}$ & 0.80 & 0.34 & 0.80 & 0.28 & 0.00 & $\mathrm{X}$ & \\
\hline KJ-1 & 0.33 & 21.29 & & & $\mathrm{X}$ & 0.77 & $\mathrm{X}$ & $\mathrm{X}$ & & & $\mathrm{X}$ & \\
\hline BJ-137-A & 0.38 & 21.67 & 0.64 & 6.04 & $\mathrm{X}$ & 0.82 & $\mathrm{X}$ & 0.74 & 0.34 & 0.02 & $\mathrm{X}$ & $\mathrm{X}$ \\
\hline JB-05 & 0.36 & 22.45 & 0.57 & 6.04 & $\mathrm{X}$ & $\mathrm{X}$ & & 0.68 & 0.39 & (na) & $\mathrm{X}$ & $\mathrm{X}$ \\
\hline XH-050-B & 0.16 & 20.89 & 0.38 & 2.55 & & & & 0.63 & 0.28 & 0.00 & $\mathrm{X}$ & \\
\hline \multirow[t]{2}{*}{ Samples } & \multicolumn{12}{|c|}{ Retrograde phases } \\
\hline & Opx2 & $\mathrm{Op} \times 2 \mathrm{Al}_{2} \mathrm{O}_{3}$ & $\mathrm{Crd} 2$ & Spl2 & Opx3 & $\mathrm{Op} \times 3 \mathrm{Al}_{2} \mathrm{O}_{3}$ & $\mathrm{Crd} 3$ & Bt2 & $\mathrm{Ti}$ & $\mathrm{F}$ & & \\
\hline $\mathrm{KJ}-3$ & & & $\mathrm{X}$ & & & & & $\mathrm{X}$ & & & & \\
\hline KJ-1 & & & 0.75 & 0.21 & & & & $\mathrm{X}$ & & & & \\
\hline BJ-137-A & 0.65 & 3.30 & $\mathrm{X}$ & & 0.61 & 3.00 & 0.67 & $X$ & & & & \\
\hline JB-05 & & & $\mathrm{X}$ & & & & & $\mathrm{X}$ & 0.30 & 0.68 & & \\
\hline XH-050-B & & & & & & & & 0.60 & 0.27 & 0.00 & & \\
\hline
\end{tabular}

All members refer to $\mathrm{X}_{\mathrm{mg}}$, except $\mathrm{Al}_{2} \mathrm{O}_{3}$ for orthopyroxene and garnet, Ti and $\mathrm{F}$ for biotite, and $\mathrm{Na}$ for plagioclase. $\mathrm{Al}_{2} \mathrm{O}_{3}$ (wt.\%), Ti, $\mathrm{F}$ and $\mathrm{Na}$ (a.p.f.u.) are average analyzed values.

from (sample JB-05) or close to (samples KJ-1, KJ-3, BJ137-A) the contact with the Brejões Dome charnockite intrusion, and from garnet-bearing charnockitic granulite (sample XH-050-B) (Fig. 2).

\subsection{Aluminous-magnesian granulites}

Samples KJ-1 and KJ-3 exhibit pervasive mylonitic foliation marked by quartz (Qtz, 35-40\%) in ribbons (up to $12.0 \mathrm{~mm}$ ) associated to elongate and almond-shaped grains of plagioclase (P11, 30-40\%), garnet (Grt1, 810\%), biotite (Bt1, 5\%), sillimanite (Sill1, 3\%), spinel (Spl1, 1-3\%) and cordierite (Crd1, 10-15\%) (Table 1). Ilmenite $(1-2 \%)$, magnetite/pyrite $(1-2 \%)$, rutile (1$2 \%$ ), graphite $(1 \%)$, monazite and zircon occur as inclusions and constitute accessory minerals. In samples BJ-137-A and JB-05, orthopyroxene (Opx1, 5\%) and rare interstitial mesoperthite $(\mathrm{Mp} 1)$ are observed in addition to the above-mentioned assemblage (Table 1).

Quartz (Qtz) occurs as oriented porhyroblasts (2.0$4.3 \mathrm{~mm})$ and xenomorphic $(0.3-1.2 \mathrm{~mm})$ crystals that display undulating extinction, deformation bands and development of sub-grains with serrated to lobate boundaries with other quartz grains. Quartz also forms microstructures which bulge into neighbouring deformed garnet porphyroblasts (Fig. 3a, b). These mylonitic microstructures attest to deformation under granulite-facies metamorphic conditions. Spinel is present as lamellae and globules (Spl1) inside cordierite, sometimes forming symplectites (sample KJ-3, Fig. 3a, b), or as idiomorphic to hypidiomorphic grains (Spl2) between 0.3 and $0.6 \mathrm{~mm}$ long in- cluded in quartz. In the latter case it may have welldeveloped crystallographic faces against quartz, as in samples KJ-1 and KJ-3 collected at the contact with CH6 charnockites of the Brejões and Santa Inês domes (Fig. 3c), which suggests equilibrium conditions, and permits to constrain minimum $P-T$ conditions for the rocks. Plagioclase (P11) forms hypidiomorphic porphyroblasts up to $3 \mathrm{~mm}$ long with quartz and biotite inclusions and polygonal aggregates of idiomorphic crystals up to $0.6 \mathrm{~mm}$ long. Most of the porphyroblasts have $\mathrm{K}$-feldspar lamellae, and are therefore antiperthitic plagioclase (Antp) with albite twinning which is sometimes bent. Garnet (Grt1) occurs as xenomorphic and hypidiomorphic crystals $(0.3-1.0 \mathrm{~mm})$, and porphyroblasts $(1.0-3.0 \mathrm{~mm})$ with straight or lobate contacts with cordierite and quartz (Fig. 3a, d). The porphyroblasts have small inclusions of quartz, plagioclase, ilmenite and biotite (Fig. 3a, b, d). Biotite (Bt1) has reddish-brown pleochroism, and occurs as very small $(0.1 \mathrm{~mm})$ inclusions within quartz, garnet and plagioclase (Fig. 3d, e), as well as oriented inclusions up to $1.0 \mathrm{~mm}$ long in orthopyroxene (Fig. 3f). Sillimanite (Sil1) occurs as small inclusions $(<0.1 \mathrm{~mm})$ in cordierite (Fig. 3a, b), or as oriented prisms in contact with quartz or biotite. Cordierite (Crd1) forms porphyroblasts and moats around garnet (Fig. $3 \mathrm{a}, \mathrm{b}$ ), in grains which reach $3.0 \mathrm{~mm}$, have polygonal and lobate contacts, and show polysynthetic twinning. Another generation of cordierite ( $\mathrm{Crd} 2)$ forms coronae around spinel in contact with quartz (Fig. 3c, g). In the first type, pinitization is absent or limited to micro-channels which cut the grains, while in the second type it is more widespread. The third type of cordierite (Crd3) occurs in 

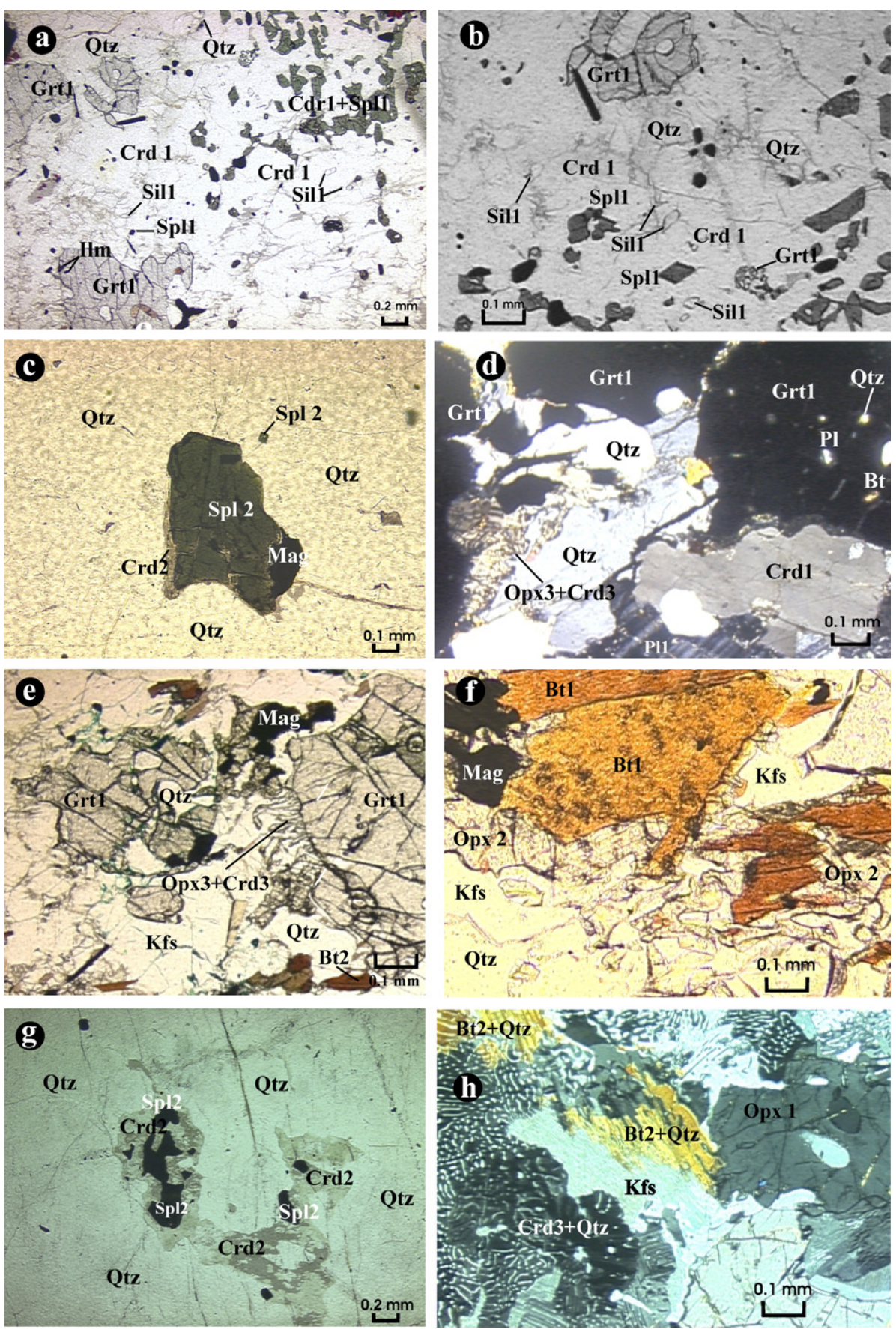

Fig. 3. (a) Crd1 + Spl1 symplectite as the product of reaction: Grt1 + Sil1 $( \pm \mathrm{Qtz})=\mathrm{Crd1}+$ Spl1. Hercynite-quartz-bearing granulite, sample KJ-3. (b) Detail of photo 3a. Please note that sillimanite and quartz were virtually consumed in the reaction Grt1 + Sil1 $( \pm \mathrm{Qtz})=\mathrm{Crd1}+$ Spl1. Hercynite-quartz-bearing granulite, sample KJ-3. (c) Hercynite crystal (Spl2) with straight borders within quartz. Hercynite-quartz-bearing granulite, sample KJ-1. (d) Biotite, quartz and plagioclase within garnet (Grt1), and reaction Grt1 $+\mathrm{Qtz}=\mathrm{Opx} 3+\mathrm{Crd} 3$. Hercynite-quartz-bearing granulite, sample KJ-1. (e) Orthopyroxene $(\mathrm{Opx} 3)+$ cordierite $(\mathrm{Crd} 3)$ symplectite formed around garnet, according to decompression reaction Grt $1+\mathrm{Qtz}=\mathrm{Opx} 3+\mathrm{Crd} 3$. Hercynite-quartz-bearing granulite, sample BJ-137-A. (f) Orthopyroxene (Opx2) + mesoperthite (Kfs) formed according to dehydration reaction $\mathrm{Bt} 1+\mathrm{Qtz}=\mathrm{Opx} 2+\mathrm{Kfs}+\mathrm{L}$. Hercynite-quartz-bearing granulite, sample BJ-137-A. (g) Hercynite crystals (Spl2) within quartz, surrounded by cordierite (Crd2) reaction coronae, according to retrograde reaction $\mathrm{Spl} 2+\mathrm{Qtz}=\mathrm{Crd} 2$. Hercynite-quartz-bearing granulite, sample KJ-1. (h) Simplectite of Bt2 + Qtz resulting from retrograde reaction Opx $1+\mathrm{Kfs}+\mathrm{L}=\mathrm{Bt} 2+\mathrm{Qtz}$. Hercynite-quartz-bearing granulite, sample KJ-3. 
Table 2

Representative chemical composition of garnet

\begin{tabular}{|c|c|c|c|c|c|c|c|c|c|c|c|c|}
\hline Samples & $\mathrm{KJ}-3$ & $\mathrm{KJ}-3$ & $\mathrm{KJ}-3$ & $\mathrm{KJ}-1$ & $\mathrm{KJ}-1$ & $\mathrm{KJ}-1$ & BJ-137-A & BJ-137-A & JB-05 & JB-05 & XH-050-B & XH-050-B \\
\hline Analysis no. & 17 & 24 & 25 & 6 & 12 & 20 & 4 & 5 & 170 & 171 & 9 & 19 \\
\hline Remarks & Rim & Core & Rim & Rim & Core & Rim & Core & Core & Core & Core & Core & Rim \\
\hline $\mathrm{SiO}_{2}$ & 38.98 & 38.89 & 38.86 & 38.25 & 38.44 & 38.59 & 38.92 & 39.09 & 38.58 & 38.35 & 37.48 & 37.97 \\
\hline $\mathrm{TiO}_{2}$ & 0.07 & 0.14 & 0.07 & 0.07 & 0.04 & 0.02 & 0.08 & 0.06 & 0.06 & 0.07 & 0.05 & 0.02 \\
\hline $\mathrm{Al}_{2} \mathrm{O}_{3}$ & 20.94 & 21.31 & 21.38 & 21.64 & 21.12 & 21.12 & 21.9 & 21.44 & 22.64 & 22.26 & 20.87 & 20.92 \\
\hline $\mathrm{Cr}_{2} \mathrm{O}_{3}$ & 0.15 & 0.08 & 0.16 & 0.09 & 0.19 & 0.13 & 0.07 & 0.09 & 0.17 & 0.23 & 0.05 & 0.00 \\
\hline $\mathrm{FeO}$ & 27.45 & 27.44 & 28.34 & 29.00 & 29.51 & 30.38 & 26.26 & 25.97 & 27.57 & 27.88 & 32.37 & 33.76 \\
\hline $\mathrm{MnO}$ & 0.47 & 0.42 & 0.52 & 1.58 & 1.88 & 1.71 & 0.30 & 0.40 & 1.32 & 1.40 & 1.13 & 1.30 \\
\hline $\mathrm{MgO}$ & 9.33 & 9.49 & 9.45 & 8.12 & 7.89 & 7.37 & 9.96 & 9.87 & 8.84 & 9.04 & 4.12 & 3.36 \\
\hline $\mathrm{CaO}$ & 1.23 & 1.6 & 1.07 & 1.23 & 1.12 & 1.24 & 1.25 & 1.25 & 1.67 & 1.23 & 2.16 & 1.97 \\
\hline $\mathrm{Na}_{2} \mathrm{O}$ & 0.05 & 0.04 & 0.00 & 0.02 & 0.00 & 0.01 & 0.01 & 0.01 & 0.01 & 0.01 & 0.02 & 0.02 \\
\hline $\mathrm{K}_{2} \mathrm{O}$ & 0.04 & 0.03 & 0.00 & 0.03 & 0.03 & 0.01 & 0.03 & 0.00 & 0.00 & 0.00 & 0.00 & 0.00 \\
\hline $\mathrm{F}$ & 0.36 & 0.00 & 0.00 & 0.09 & 0.00 & 0.00 & 0.31 & 0.36 & 0.00 & 0.00 & 0.00 & 0.00 \\
\hline Sum & 99.05 & 99.44 & 99.85 & 100.12 & 100.22 & 100.58 & 99.09 & 98.24 & 100.86 & 100.47 & 98.25 & 99.32 \\
\hline
\end{tabular}

Structural formulae based on 12 oxygens

\begin{tabular}{|c|c|c|c|c|c|c|c|c|c|c|c|c|}
\hline $\mathrm{Si}$ & 2.99 & 3.01 & 3.00 & 2.97 & 2.99 & 3.00 & 3.06 & 3.05 & 2.99 & 2.99 & 3.04 & 3.02 \\
\hline $\mathrm{Al}^{\mathrm{IV}}$ & 0.01 & 0.00 & 0.00 & 0.03 & 0.01 & 0.00 & 0.00 & 0.00 & 0.01 & 0.01 & 0.00 & 0.00 \\
\hline $\mathrm{Al}^{\mathrm{VI}}$ & 1.94 & 1.94 & 1.95 & 1.95 & 1.93 & 1.94 & 1.98 & 1.97 & 2.02 & 1.99 & 1.99 & 2.01 \\
\hline $\mathrm{Ti}$ & 0.01 & 0.01 & 0.01 & 0.01 & 0.01 & 0.01 & 0.01 & 0.01 & 0.00 & 0.00 & 0.01 & 0.00 \\
\hline $\mathrm{Cr}$ & 0.01 & 0.01 & 0.01 & 0.01 & 0.01 & 0.01 & 0.01 & 0.01 & 0.01 & 0.01 & 0.01 & 0.00 \\
\hline $\mathrm{Fe}^{3+}$ & 0.06 & 0.03 & 0.03 & 0.06 & 0.06 & 0.05 & 0.00 & 0.00 & 0.00 & 0.00 & 0.00 & 0.00 \\
\hline $\mathrm{Fe}^{2+}$ & 1.76 & 1.74 & 1.79 & 1.82 & 1.85 & 1.93 & 1.68 & 1.68 & 1.74 & 1.77 & 2.19 & 2.30 \\
\hline $\mathrm{Mn}$ & 0.04 & 0.03 & 0.03 & 0.10 & 0.12 & 0.11 & 0.02 & 0.02 & 0.08 & 0.09 & 0.07 & 0.06 \\
\hline $\mathrm{Mg}$ & 1.09 & 1.09 & 1.09 & 0.94 & 0.91 & 0.85 & 1.14 & 1.15 & 0.99 & 1.02 & 0.49 & 0.41 \\
\hline $\mathrm{Ca}$ & 0.10 & 0.13 & 0.09 & 0.10 & 0.09 & 0.10 & 0.10 & 0.10 & 0.13 & 0.10 & 0.18 & 0.17 \\
\hline $\mathrm{Na}$ & 0.01 & 0.01 & 0.00 & 0.01 & 0.00 & 0.01 & 0.00 & 0.00 & 0.00 & 0.00 & 0.00 & 0.01 \\
\hline $\mathrm{K}$ & 0.01 & 0.01 & 0.00 & 0.01 & 0.01 & 0.01 & 0.00 & 0.00 & 0.00 & 0.00 & 0.00 & 0.00 \\
\hline$X_{M g}$ & 0.38 & 0.38 & 0.37 & 0.34 & 0.33 & 0.31 & 0.38 & 0.38 & 0.38 & 0.34 & 0.18 & 0.15 \\
\hline $\mathrm{Al}$ & 74.00 & 68.70 & 68.00 & 61.30 & 62.10 & 64.30 & 57.10 & 56.70 & 58.90 & 59.30 & 74.10 & 78.00 \\
\hline Py & 16.70 & 23.60 & 23.80 & 31.70 & 30.60 & 28.50 & 38.60 & 38.80 & 33.60 & 34.30 & 16.80 & 13.80 \\
\hline $\mathrm{Sp}$ & 2.80 & 4.40 & 4.60 & 3.50 & 4.10 & 3.80 & 0.70 & 0.90 & 2.90 & 3.00 & 2.60 & 2.30 \\
\hline $\mathrm{Gr}$ & 6.30 & 1.30 & 0.40 & 0.00 & 0.00 & 0.50 & 3.00 & 3.00 & 3.90 & 2.40 & 6.00 & 5.70 \\
\hline Ad & 0.00 & 1.00 & 2.80 & 3.40 & 3.10 & 2.40 & 0.00 & 0.00 & 0.00 & 0.00 & 0.00 & 0.00 \\
\hline UV & 0.20 & 0.70 & 0.40 & 0.10 & 0.00 & 0.40 & 0.20 & 0.30 & 0.50 & 0.70 & 0.02 & 0.00 \\
\hline
\end{tabular}

$\mathrm{XH}-050-\mathrm{B}$ is garnet-bearing charnockitic granulite sample and the others are aluminous-magnesian granulites.

symplectites with orthopyroxene (Opx3) around garnet (Grt1) (Fig. 3d, e) or in symplectites with quartz close to orthopyroxene porphyroblasts (Opx1) (Fig. 3h). Orthopyroxene (Opx1) occurs as oriented xenomorphic to hypidiomorphic crystals with dimensions of $0.3-1.0 \mathrm{~mm}$ (Fig. 3h), some of them with biotite inclusions. Orthopyroxene also forms symplectitic coronae (Opx2) with $\mathrm{K}$-feldspar (Kfs) surrounding biotite (Bt1) (Fig. 3f) in sample BJ-137-A at the contact with the charnockite domes. A third generation of orthopyroxene occurs as lamellae and globules (Opx3) in cordierite-host symplectites (Fig. 3d, e).

In relation to the accessory phases only magnetite appears in modal proportion greater than $1 \%$. It is in contact with spinel, garnet and biotite (Fig. 3c, e, f). Additional opaque minerals (ilmenite, graphite) are scattered throughout the rock or sometimes included in garnet.
Monazite and zircon are readily recognized as inclusions in cordierite and biotite due to the yellowish pleochroic haloes produced in their host-grains.

Microstructural relationships indicate that the peak metamorphic mineral paragenesis of the aluminousmagnesian granulites is composed of $\mathrm{Qtz}+\mathrm{Spl1}+\mathrm{P} 11+$ Grt1+Sil1 + Bt1. Retrograde granulite-metamorphic paragenesis are seen in interstitial positions between peak metamorphic minerals and they are formed of: (i) a second generation of cordierite $(\mathrm{Crd} 2)$ at the contact between hercynite and quartz (Fig. 3c); (ii) symplectitic coronae of cordierite (Crd3) with a third generation of orthopyroxene (Opx3) at the contact between garnet and quartz (Fig. 3d, e); (iii) a second type of biotite $\left(\mathrm{Bt}_{2}\right)$ constitutes intergrowths with quartz in coronae around orthopyroxene (Fig. 3h) and opaque minerals. 
Table 3

Representative chemical composition of orthopyroxene

\begin{tabular}{|c|c|c|c|c|c|c|c|c|c|}
\hline Samples & BJ-137 A & BJ-137 A & BJ-137 A & BJ-137-A & BJ-137-A & JB-05 & XH-050-B & XH-050-B & XH-050-B \\
\hline Analysis no. & 2 & 3 & 11 & 14 & 17 & 1 & 1 & 2 & 4 \\
\hline Remarks & Opx1(Core) & Opx1(Core) & Opx2(Core) & Opx3(Core) & Opx3(Rim) & Opx1(Core) & Opx1(Core) & Opx1(Core) & Opx1(Rim) \\
\hline $\mathrm{SiO}_{2}$ & 48.96 & 49.4 & 50.91 & 50.92 & 50.93 & 48.77 & 47.84 & 48.17 & 48.34 \\
\hline $\mathrm{TiO}_{2}$ & 0.13 & 0.18 & 0.04 & 0.14 & 0.15 & 0.15 & 0.15 & 0.13 & 0.16 \\
\hline $\mathrm{Al}_{2} \mathrm{O}_{3}$ & 5.67 & 6.42 & 3.30 & 3.00 & 3.00 & 6.04 & 2.60 & 2.63 & 2.44 \\
\hline $\mathrm{Cr}_{2} \mathrm{O}_{3}$ & 0.19 & 0.04 & 0.00 & 0.39 & 0.40 & 0.26 & 0.00 & 0.01 & 0.00 \\
\hline $\mathrm{FeO}$ & 21.33 & 20.90 & 22.03 & 22.82 & 22.99 & 26.17 & 35.02 & 34.65 & 34.4 \\
\hline $\mathrm{MnO}$ & 0.22 & 0.06 & 0.30 & 0.39 & 0.40 & 0.43 & 0.3 & 0.35 & 0.35 \\
\hline $\mathrm{MgO}$ & 20.86 & 21.48 & 22.10 & 20.05 & 19.93 & 18.52 & 12.23 & 12.44 & 13.11 \\
\hline $\mathrm{CaO}$ & 0.19 & 0.11 & 0.09 & 0.10 & 0.09 & 0.10 & 0.32 & 0.30 & 0.26 \\
\hline $\mathrm{Na}_{2} \mathrm{O}$ & 0.02 & 0.03 & 0.04 & 0.06 & 0.07 & 0.01 & 0.02 & 0.03 & 0.00 \\
\hline $\mathrm{K}_{2} \mathrm{O}$ & 0.00 & 0.00 & 0.01 & 0.04 & 0.04 & 0.00 & 0.00 & 0.03 & 0.01 \\
\hline $\mathrm{F}$ & 0.04 & 0.16 & 0.00 & 0.27 & 0.20 & & & & \\
\hline Sum & 97.61 & 98.78 & 98.82 & 98.18 & 98.20 & 100.45 & 98.48 & 98.74 & 99.07 \\
\hline \multicolumn{10}{|c|}{ Structural formulae based on 12 oxygens } \\
\hline $\mathrm{Si}$ & 1.86 & 1.84 & 1.91 & 1.94 & 1.91 & 0.16 & 1.93 & 1.93 & 1.95 \\
\hline $\mathrm{Al}^{\mathrm{IV}}$ & 0.13 & 0.15 & 0.09 & 0.05 & 0.09 & 0.10 & 0.07 & 0.06 & 0.05 \\
\hline $\mathrm{Al}^{\mathrm{VI}}$ & 0.12 & 0.13 & 0.05 & 0.10 & 0.07 & 0.01 & 0.05 & 0.06 & 0.02 \\
\hline $\mathrm{Ti}$ & 0.00 & 0.00 & 0.00 & 0.00 & 0.01 & 0.01 & 0.00 & 0.00 & 0.01 \\
\hline $\mathrm{Cr}$ & 0.00 & 0.00 & 0.00 & 0.00 & 0.01 & 0.04 & 0.00 & 0.00 & 0.00 \\
\hline $\mathrm{Fe}^{3+}$ & 0.00 & 0.01 & 0.03 & 0.00 & 0.01 & 0.01 & 0.01 & 0.00 & 0.02 \\
\hline $\mathrm{Fe}^{2+}$ & 0.67 & 0.64 & 0.65 & 0.72 & 0.72 & 0.78 & 1.17 & 1.16 & 1.14 \\
\hline $\mathrm{Mn}$ & 0.00 & 0.00 & 0.01 & 0.00 & 0.01 & 0.01 & 0.01 & 0.01 & 0.01 \\
\hline $\mathrm{Mg}$ & 1.18 & 1.19 & 1.23 & 1.15 & 0.16 & 1.04 & 0.73 & 0.74 & 0.79 \\
\hline $\mathrm{Ca}$ & 0.00 & 0.00 & 0.00 & 0.00 & 0.00 & 0.00 & 0.01 & 0.01 & 0.01 \\
\hline $\mathrm{Na}$ & 0.00 & 0.00 & 0.01 & 0.00 & 0.00 & 0.00 & 0.00 & 0.00 & 0.00 \\
\hline $\mathrm{K}$ & 0.00 & 0.00 & 0.00 & 0.00 & 0.00 & 0.00 & 0.00 & 0.00 & 0.00 \\
\hline $\mathrm{F}$ & 0.00 & 0.00 & 0.00 & 0.00 & 0.00 & 0.00 & 0.00 & 0.00 & 0.00 \\
\hline $\mathrm{X}_{\mathrm{Mg}}$ & 0.64 & 0.65 & 0.65 & 0.61 & 0.61 & 0.57 & 0.38 & 0.39 & 0.52 \\
\hline
\end{tabular}

$\mathrm{XH}-050-\mathrm{B}$ is garnet-bearing charnockitic granulite sample and the others are aluminous-magnesian granulites.

\subsection{Garnet-bearing charnockitic granulite}

Sample XH-050-B is constituted of mesoperthite (Mp1, 40-50\%), quartz (Qtz, 30\%), antiperthitic plagioclase (P11, 10\%), orthopyroxene (Opx1, 10\%), sometimes transformed into bastite, garnet (Grt1, 5-10\%) and biotite (Bt1, 2-5\%). Mesoperthite (Mp1) occurs as porphyroblasts $(0.8-3.0 \mathrm{~mm})$ with lobate to straight contacts with garnet and plagioclase. Myrmekite is commonly developed at its contact with plagioclase. Quartz (Qtz1) occurs as xenomorphic porphyroblasts $(1.0-2.5 \mathrm{~mm})$ that are fractured and exhibit strong undulating extinction and deformation bands. The porphyroblasts show lobate boundaries against the other grains. Antiperthitic plagioclase (Pl1) forms prismatic crystals $(0.6-3.0 \mathrm{~mm})$ with curved albite twins. The larger crystals contain inclusions of garnet and biotite and show $\mathrm{K}$-feldspar lamellae. Orthopyroxene (Opx1) constitutes xenomorphic crystals $(<0.5 \mathrm{~mm})$, most of them altered to bastite. Orthopyroxene (Opx1) also forms coronae together with plagioclase (P11) around garnet megacrystals. Garnet (Grt1) forms idio- morphic to hypidiomorphic porphyroblasts, 0.7 to $2.5 \mathrm{~mm}$ long, with borders resorbed by mesoperthite, plagioclase and quartz. The porphyroblasts contain inclusions of plagioclase, biotite and quartz. Biotite (Bt1) occurs as dark-red porphyroblasts that are dispersed among the other main mineral phases. Its cleavage is always oriented parallel to the rock foliation. A second generation of biotite (Bt2) is formed from orthopyroxene and garnet, and occurs in biotite-quartz symplectites, as well as flakes also oriented parallel to the rock foliation. Ilmenite, pyrite, apatite, zircon, and monazite are accessory phases. Myrmekite is also found along contacts between plagioclase, mesoperthite and quartz in these metaplutonic rocks re-equilibrated in granulite facies.

\section{Mineral chemistry}

\subsection{Analytical methods}

Mineral compositions were obtained at Paris VI University, France using the Camebax SX 100 electron 
microprobe. Operating conditions were $15 \mathrm{kV}$ accelerating voltage and $10 \mathrm{nA}$ specimen current and 1-2 $\mu \mathrm{m}$ beam diameter. Natural silicates and synthetic oxides were used as standards for all elements. Representative analytical results of the main mineral phases of garnetbearing charnockitic granulite (HG) and aluminousmagnesian granulite samples (SP) are shown in Tables 17. Structural formulae were calculated with the Norm Version 4.0 Program of Ulm (1993). Abbreviations follow Kretz (1983), plus Mp for mesoperthite.

\subsection{Aluminous-magnesian granulites}

Garnet from aluminous-magnesian granulites displays small chemical variations among grains. It is relatively rich in the almandine component (about $60 \%$ ), but it is richer in pyrope component (around 30$35 \%$ ) than garnet (Grt1) crystals from the garnet-bearing charnockitic granulite. The grossular and spessartite end- members are present in low concentrations $(<5 \%)$ (Fig. 4 , Table 2). Microprobe profiles across two garnet porphyroblasts from samples KJ-1 and KJ-3 reveal slight zoning of iron and magnesium contents. In the garnet porphyroblast from sample KJ-1 there is iron enrichment and magnesium depletion from core to rim, due to the take-up of $\mathrm{Mg}$ in the formation of the cordierite envelope. $\mathrm{Mg}$ and Fe zoning in the garnet porphyroblast from sample KJ-3 repeats this pattern. Ca is richer in the core than at the rim. This feature can be attributed to the formation of plagioclase around garnet, in which plagioclase took up $\mathrm{Ca}$ from the garnet rims.

Orthopyroxene in sample BJ-137-A occurs in three generations that are different in chemistry: Opx 1 is richer in aluminium $\left(\approx 6.0 \mathrm{wt} . \% \mathrm{Al}_{2} \mathrm{O}_{3}\right)$ than $\mathrm{Opx} 2(\approx 3.5 \mathrm{wt} . \%$ $\mathrm{Al}_{2} \mathrm{O}_{3}$ ), while $\mathrm{Opx} 3$ with $\approx 3.0$ wt. $\% \mathrm{Al}_{2} \mathrm{O}_{3}$ has higher contents of $\mathrm{Fe}$ than Opx1 and Opx2 (Fig. 5, Table 3). The low $\mathrm{Al}_{2} \mathrm{O}_{3}$ concentrations of around $6.0 \mathrm{wt} . \%$ in Opx1 stand out, being lower than those found in other

Table 4

Representative chemical composition of biotite

\begin{tabular}{|c|c|c|c|c|c|c|c|c|}
\hline Samples & $\mathrm{KJ}-3$ & $\mathrm{KJ}-3$ & BJ-137-A & BJ-137-A & JB-05 & JB-05 & XH-050-B & XH-050-B \\
\hline Analysis no. & 29 & 30 & 6 & 16 & 173 & 172 & 18 & 20 \\
\hline Remarks & Bt1(Core) & Bt1(Core) & Bt1(Core) & Bt1(Core) & Bt1(Core) & $\mathrm{Bt} 2$ (Core) & Bt1(Core) & Bt2(Core) \\
\hline $\mathrm{SiO}_{2}$ & 37.59 & 37.27 & 37.98 & 37.88 & 37.24 & 39.15 & 37.16 & 38.47 \\
\hline $\mathrm{TiO}_{2}$ & 5.12 & 5.73 & 5.13 & 6.61 & 4.50 & 3.94 & 4.84 & 4.58 \\
\hline $\mathrm{Al}_{2} \mathrm{O}_{3}$ & 15.9 & 15.55 & 14.98 & 14.23 & 15.65 & 16.63 & 13.36 & 12.38 \\
\hline $\mathrm{Cr}_{2} \mathrm{O}_{3}$ & 0.30 & 0.16 & 0.28 & 0.35 & 0.51 & 0.41 & 0.01 & 0.08 \\
\hline $\mathrm{FeO}$ & 7.53 & 7.97 & 7.04 & 11.37 & 15.32 & 9.15 & 14.91 & 16.56 \\
\hline $\mathrm{MnO}$ & 0.00 & 0.05 & 0.00 & 0.27 & 0.02 & 0.03 & 0.14 & 0.00 \\
\hline $\mathrm{MgO}$ & 17.86 & 17.62 & 17.84 & 13.72 & 14.98 & 18.31 & 14.11 & 13.53 \\
\hline $\mathrm{CaO}$ & 0.03 & 0.00 & 0.03 & 0.00 & 0.04 & 0.02 & 0.00 & 0.00 \\
\hline $\mathrm{Na}_{2} \mathrm{O}$ & 0.42 & 0.37 & 0.42 & 0.05 & 0.12 & 0.08 & 0.12 & 0.09 \\
\hline $\mathrm{K}_{2} \mathrm{O}$ & 9.31 & 9.52 & 8.93 & 9.51 & 9.35 & 9.27 & 9.77 & 9.80 \\
\hline $\mathrm{H}_{2} \mathrm{O}$ & 2.98 & 3.26 & 3.29 & 3.63 & 2.04 & 2.11 & 3.99 & 3.56 \\
\hline $\mathrm{F}$ & 2.39 & 1.76 & 2.44 & 2.63 & & & 0.00 & 0.00 \\
\hline Sum & 99.56 & 99.38 & 98.36 & 100.25 & 99.8 & 99.1 & 98.41 & 99.05 \\
\hline \multicolumn{9}{|c|}{ Structural formulae based on 11 oxygens } \\
\hline $\mathrm{Si}$ & 2.65 & 2.63 & 2.80 & 2.86 & 2.73 & 2.82 & 2.88 & 2.92 \\
\hline $\mathrm{Al}^{\mathrm{IV}}$ & 1.22 & 1.19 & 1.19 & 1.14 & 1.26 & 1.17 & 1.11 & 1.07 \\
\hline $\mathrm{Al}^{\mathrm{VI}}$ & 0.05 & 0.10 & 0.17 & 0.19 & 0.09 & 0.24 & 0.11 & 0.16 \\
\hline $\mathrm{Ti}$ & 0.27 & 0.30 & 0.30 & 0.39 & 0.39 & 0.30 & 0.28 & 0.27 \\
\hline $\mathrm{Cr}$ & 0.01 & 0.01 & 0.02 & 0.02 & 0.02 & 0.01 & 0.00 & 0.01 \\
\hline $\mathrm{Fe}^{3+}$ & 0.00 & 0.00 & 0.00 & 0.00 & 0.00 & 0.00 & 0.00 & 0.00 \\
\hline $\mathrm{Fe}^{2+}$ & 0.44 & 0.47 & 0.45 & 0.75 & 1.06 & 0.55 & 0.97 & 1.01 \\
\hline $\mathrm{Mn}$ & 0.00 & 0.01 & 0.00 & 0.02 & 0.00 & 0.00 & 0.01 & 0.00 \\
\hline $\mathrm{Mg}$ & 1.88 & 1.85 & 2.06 & 1.62 & 1.48 & 1.97 & 1.63 & 1.55 \\
\hline $\mathrm{Ca}$ & 0.01 & 0.00 & 0.00 & 0.00 & 0.05 & 0.00 & 0.00 & 0.00 \\
\hline $\mathrm{Na}$ & 0.06 & 0.05 & 0.06 & 0.01 & 0.01 & 0.06 & 0.02 & 0.01 \\
\hline $\mathrm{K}$ & 0.84 & 0.85 & 0.88 & 0.96 & 0.96 & 0.88 & 0.97 & 0.97 \\
\hline F & 0.00 & 0.00 & 0.02 & 0.02 & 0.30 & 0.68 & 0.00 & 0.00 \\
\hline $\mathrm{OH}$ & 0.74 & 0.81 & 2.00 & 2.00 & 2.00 & 2.00 & 2.00 & 2.00 \\
\hline $\mathrm{X}_{\mathrm{Mg}}$ & 0.81 & 0.80 & 0.81 & 0.68 & 0.58 & 0.78 & 0.63 & 0.60 \\
\hline
\end{tabular}

$\mathrm{XH}-050-\mathrm{B}$ is garnet-bearing charnockitic granulite sample and the others are aluminous-magnesian granulites. 
Table 5

Representative chemical composition of cordierite and sillimanite

\begin{tabular}{|c|c|c|c|c|c|c|c|c|c|}
\hline Samples & $\mathrm{KJ}-3$ & $\mathrm{KJ}-3$ & $\mathrm{KJ}-1$ & $\mathrm{KJ}-1$ & $\mathrm{KJ}-1$ & BJ-137-A & BJ-137-A & BJ-137-A & $\mathrm{KJ}-3$ \\
\hline Analysis no. & 13 & 14 & 31 & 32 & 36 & 19 & 9 & 13 & 1 \\
\hline Remarks & Crd1(Core) & Crd1(Core) & Crd1(Rim) & Crd1(Rim) & $\mathrm{Crd} 2$ (around $\mathrm{Hc}$ ) & Crd1(Core) & $\mathrm{Crd} 3 / \mathrm{Opx} 3$ & $\mathrm{Crd} 3 / \mathrm{Opx} 3$ & Sill \\
\hline $\mathrm{SiO}_{2}$ & 48.97 & 49.34 & 49.37 & 49.42 & 48.79 & 48.81 & 49.83 & 49.83 & 36.65 \\
\hline $\mathrm{TiO}_{2}$ & 0.00 & 0.01 & 0.02 & 0.01 & 0.00 & 0.00 & 0.00 & 0.00 & 0.00 \\
\hline $\mathrm{Al}_{2} \mathrm{O}_{3}$ & 33.15 & 32.75 & 33.36 & 33.56 & 33.53 & 32.42 & 33.30 & 33.03 & 61.12 \\
\hline $\mathrm{Cr}_{2} \mathrm{O}_{3}$ & 0.05 & 0.07 & 0.04 & 0.15 & 0.00 & 0.12 & 0.00 & 0.00 & 0.08 \\
\hline $\mathrm{FeO}$ & 4.91 & 4.99 & 5.32 & 5.31 & 6.07 & 4.02 & 4.70 & 4.91 & 0.21 \\
\hline $\mathrm{MnO}$ & 0.10 & 0.00 & 0.00 & 0.15 & 0.10 & 0.01 & 0.00 & 0.00 & 0.00 \\
\hline $\mathrm{MgO}$ & 10.90 & 10.71 & 10.25 & 9.98 & 9.86 & 10.76 & 9.80 & 10.15 & 0.04 \\
\hline $\mathrm{CaO}$ & 0.00 & 0.00 & 0.00 & 0.00 & 0.03 & 0.00 & 0.01 & 0.01 & 0.02 \\
\hline $\mathrm{Na}_{2} \mathrm{O}$ & 0.03 & 0.03 & 0.13 & 0.11 & 0.09 & 0.06 & 0.06 & 0.05 & 0.03 \\
\hline $\mathrm{K}_{2} \mathrm{O}$ & 0.00 & 0.01 & 0.01 & 0.03 & 0.02 & 0.03 & 0.00 & 0.00 & 0.01 \\
\hline $\mathrm{F}$ & 0.00 & 0.29 & 0.07 & 0.00 & 0.14 & 0.14 & 0.09 & 0.09 & 0.00 \\
\hline Sum & 98.11 & 98.2 & 98.57 & 98.72 & 98.63 & 96.37 & 97.79 & 98.07 & 98.16 \\
\hline \multicolumn{10}{|c|}{ Structural formulae based on 18 oxygens (Crd); 20 oxygens (Sil) } \\
\hline $\mathrm{Si}$ & 4.97 & 5.02 & 5.00 & 4.99 & 4.96 & 5.03 & 5.04 & 4.99 & 4.03 \\
\hline $\mathrm{Al}$ & 3.97 & 3.93 & 3.98 & 4.00 & 4.02 & 3.94 & 3.97 & 3.98 & 7.93 \\
\hline $\mathrm{Ti}$ & 0.00 & 0.00 & 0.00 & 0.00 & 0.00 & 0.00 & 0.00 & 0.00 & 0.00 \\
\hline $\mathrm{Cr}$ & 0.01 & 0.01 & 0.00 & 0.01 & 0.00 & 0.01 & 0.00 & 0.00 & 0.01 \\
\hline $\mathrm{Fe}^{2+}$ & 0.42 & 0.42 & 0.45 & 0.45 & 0.51 & 0.35 & 0.62 & 0.52 & 0.02 \\
\hline $\mathrm{Mn}$ & 0.01 & 0.00 & 0.00 & 0.01 & 0.01 & 0.00 & 0.00 & 0.00 & 0.00 \\
\hline $\mathrm{Mg}$ & 1.65 & 1.63 & 1.55 & 1.50 & 1.49 & 1.65 & 1.20 & 1.21 & 0.01 \\
\hline $\mathrm{Ca}$ & 0.00 & 0.00 & 0.00 & 0.00 & 0.01 & 0.00 & 0.00 & 0.00 & 0.00 \\
\hline $\mathrm{Na}$ & 0.01 & 0.01 & 0.02 & 0.02 & 0.02 & 0.01 & 0.01 & 0.01 & 0.01 \\
\hline $\mathrm{K}$ & 0.00 & 0.00 & 0.00 & 0.00 & 0.00 & 0.01 & 0.00 & 0.00 & 0.00 \\
\hline $\mathrm{F}$ & 0.00 & 0.09 & 0.02 & 0.00 & 0.04 & 0.00 & 0.00 & 0.00 & 0.00 \\
\hline $\mathrm{X}_{\mathrm{Mg}}$ & 0.80 & 0.80 & 0.77 & 0.77 & 0.75 & 0.82 & 0.66 & 0.67 & 0.33 \\
\hline
\end{tabular}

All samples are aluminous-magnesian granulites.

aluminous-magnesian granulites in the rest of the world, such as those of the Central Limpopo Belt, which have $\mathrm{Al}_{2} \mathrm{O}_{3}$ contents around 8.0 wt.\% (Harris and Holland, 1984a,b), those of North-Central Madagascar with up to 9.0 wt. $\%$ de $\mathrm{Al}_{2} \mathrm{O}_{3}$ (Gonçalves et al., 2004), and those of the Central Highland Complex whose orthoyroxene contains between 8.0 and 12.5 wt. $\% \mathrm{Al}_{2} \mathrm{O}_{3}$ (Sajeev and Osanai, 2004). The $\mathrm{Al}_{2} \mathrm{O}_{3}$ contents in orthopyroxene seem to reflect those of the metapelite protholiths which are poor in alumina in the studied region (Barbosa, 1986). The molar concentrations of magnesium in the studied orthopyroxene $\left(\mathrm{X}_{\mathrm{Mg}}=0.60\right)$ are also lower than those in the three cited areas, which lie between 0.70 and 0.80 .

Biotite occurs in two distinct generations (Bt1, Bt2) in samples KJ-3, BJ-137-A and JB-5. Peak/close-to-peak biotite (Bt1) is richer in magnesium $\left(\mathrm{X}_{\mathrm{Mg}} \approx 0.81\right)$ than retrograde biotite $\left(\mathrm{Bt} 2 \mathrm{X}_{\mathrm{Mg}} \approx 0.78\right)$ in symplectites with quartz (Tables 1, 4). Using the classification of Hormann et al. (1980), Bt1 is phlogopite-meroxene, whereas Bt2 is meroxene. Titanium contents of Bt1 vary between $5 \%$ and $6 \%$ in samples $\mathrm{KJ}-3, \mathrm{BJ}-137-\mathrm{A}$ from close to the Brejões Dome to around $4 \%$ in sample JB-5, away from the dome. Fluorine contents are also higher (1.762.63 wt.\%) in Bt1 than in Bt2, which has insignificant or zero concentrations.

Cordierite from different samples (Tables 1, 5) is present in three generations, Crd1, Crd2, and Crd3, which have decreasing $\mathrm{X}_{\mathrm{Mg}}$ ratios, in $\mathrm{Crd} 1$ between 0.77 and 0.82, in Crd2 around 0.75, and in Crd3, 0.66-0.67. The high wt.\% totals in the range 96.37-98.7 indicate that only small quantities of fluid species such as $\mathrm{H}_{2} \mathrm{O}$ and $\mathrm{CO}_{2}$ are present in the structural channels.

Spinel occurs in symplectites with cordierite (Spl1), or in direct contact with quartz (Sp12). The two types show important differences in composition (Fig. 6, Tables 1, 6). Both spinel generations are hercynite-rich solid solutions, with $\mathrm{X}_{\mathrm{Mg}}$ ratios varying from 0.33 to 0.34 for Spl1, and from 0.19 to 0.23 for $\mathrm{Spl} 2 . \mathrm{Cr}_{2} \mathrm{O}_{3}$ contents vary from 0.14 to $0.15 \mathrm{wt} . \%$ for Spl1, and from 2.32 to $4.73 \mathrm{wt} \%$ for $\mathrm{Spl} 2$. $\mathrm{ZnO}$ contents vary from 0.76 to $0.82 \mathrm{wt} . \%$ for Spl1, and from 1.96 to $2.75 \mathrm{wt} . \%$ for Spl2.

Plagioclase compositions are variable even in the aluminous-magnesian granulites. In KJ-3 the Ab content lies between 61 and $66 \mathrm{wt} . \%$, while in JB-5 it is around 
50 wt.\%. The potash feldspar is essentially orthoclase (Table 7). Ilmenite has $\mathrm{TiO}_{2}$ contents of ca. $54 \%$ and $\mathrm{FeO}$ contents of ca. $46 \%$. Magnetite, often associated with spinel, has an $\mathrm{X}_{\mathrm{Fe}}$ ratio $\left(\mathrm{Fe}^{+3} / \mathrm{Fe}^{+3}+\mathrm{Fe}^{+2}\right)$ of 0.66 .

\subsection{Garnet-bearing charnockitic granulite}

Garnet (Grt1) grains are unzoned and rich in almandine (74-75\%) and pyrope (12-16\%), and poor in spessartite (2.5-3\%) (Fig. 4, Table 2). The almandine component is more abundant than in garnet from the aluminousmagnesian granulites, in which pyrope and spessartine are more abundant.

Orthopyroxene (Opx1) is ferrosilite-rich $\left(\mathrm{X}_{\mathrm{Mg}}=0.38\right.$, Fig. 5, Table 3) and it appears as $2-5 \mathrm{~mm}$ porphyroblastic crystals with biotite and opaque mineral inclusions. The aluminium contents $(\approx 2.6 \mathrm{wt} . \%)$ are significantly lower than those found in the aluminous-magnesian granulites $(\approx 6.0$ wt. $\%)$.
Biotite (Bt1) is richer in $\mathrm{Fe}(15-16$ wt.\%) than biotite in aluminous - magnesian granulites $(\approx 7-11 \mathrm{wt} . \%)$, while $\mathrm{Al}_{2} \mathrm{O}_{3}\left(\approx 13\right.$ wt.\%) and $\mathrm{TiO}_{2}(4.6-4.8$ wt.\%) contents are lower (Table 4). Antiperthitic plagioclase (P11) and mesoperthite (Mp1) are andesine and orthoclase, respectively (Table 7).

\section{Evolution of mineral assemblages}

\subsection{Aluminous-magnesian granulites}

In the aluminous-magnesian granulites most of the peak metamorphic phases $(\mathrm{Opx} 1+\mathrm{Bt} 1+\mathrm{Grt1}+\mathrm{Sill}+\mathrm{Qtz})$ are partially replaced. Greenish spots, oriented parallel to banding or foliation, are found in several outcrops. They are composed of cordierite (Crd1) plus spinel (Spl1) symplectites which surround corroded garnet grains. These microstructures are similar to those described in rocks from Forefinger Point, Napier Complex in Antarctica (Harley

Table 6

Representative chemical composition of spinel

\begin{tabular}{|c|c|c|c|c|c|c|c|c|}
\hline Samples & $\mathrm{KJ}-3$ & $\mathrm{KJ}-3$ & $\mathrm{KJ}-3$ & KJ-1 & $\mathrm{KJ}-1$ & $\mathrm{KJ}-1$ & $\mathrm{KJ}-1$ & $\mathrm{KJ}-1$ \\
\hline Analysis no. & 3 & 5 & 7 & 1 & 2 & 34 & 43 & 45 \\
\hline Remarks & $\begin{array}{l}\text { Spl1/Crd1 } \\
\text { (Core) }\end{array}$ & $\begin{array}{l}\text { Spl1/Crd1 } \\
\text { (Core) }\end{array}$ & $\begin{array}{l}\text { Spl1/Crd1 } \\
\text { (Core) }\end{array}$ & $\begin{array}{l}\mathrm{Sp} 12 / \mathrm{Qz} \\
\text { (Core) }\end{array}$ & $\begin{array}{l}\mathrm{Spl} 2 / \mathrm{Qz} \\
\text { (Core) }\end{array}$ & $\begin{array}{l}\mathrm{Spl} 2 / \mathrm{Qz} \\
\text { (Core) }\end{array}$ & $\begin{array}{l}\mathrm{Spl} 12 / \mathrm{Qz} \\
\text { (Core) }\end{array}$ & $\begin{array}{l}\mathrm{Sp} 12 / \mathrm{Qz} \\
\text { (Rim) }\end{array}$ \\
\hline $\mathrm{SiO}_{2}$ & 0.00 & 0.03 & 0.00 & 0.00 & 0.03 & 0.00 & 0.02 & 0.03 \\
\hline $\mathrm{TiO}_{2}$ & 0.02 & 0.05 & 0.09 & 0.01 & 0.05 & 0.06 & 0.03 & 0.00 \\
\hline $\mathrm{Al}_{2} \mathrm{O}_{3}$ & 59.16 & 59.18 & 59.42 & 54.75 & 55.48 & 51.27 & 55.36 & 54.81 \\
\hline $\mathrm{Cr}_{2} \mathrm{O}_{3}$ & 0.15 & 0.15 & 0.14 & 2.68 & 2.45 & 4.73 & 2.32 & 2.97 \\
\hline $\mathrm{FeO}$ & 30.58 & 30.7 & 29.46 & 34.03 & 34.03 & 35.45 & 34.00 & 34.13 \\
\hline $\mathrm{MnO}$ & 0.10 & 0.04 & 0.06 & 0.23 & 0.16 & 0.23 & 0.30 & 0.23 \\
\hline $\mathrm{MgO}$ & 8.08 & 8.21 & 8.25 & 5.33 & 5.20 & 4.13 & 4.94 & 4.75 \\
\hline $\mathrm{CaO}$ & 0.00 & 0.00 & 0.00 & 0.00 & 0.00 & 0.01 & 0.06 & 0.00 \\
\hline $\mathrm{Na}_{2} \mathrm{O}$ & 0.03 & 0.03 & 0.05 & 0.06 & 0.03 & 0.11 & 0.07 & 0.05 \\
\hline $\mathrm{K}_{2} \mathrm{O}$ & 0.00 & 0.00 & 0.01 & 0.01 & 0.00 & 0.00 & 0.02 & 0.00 \\
\hline $\mathrm{ZnO}$ & 0.80 & 0.82 & 0.76 & 2.07 & 1.96 & 2.75 & 2.15 & 2.09 \\
\hline $\mathrm{F}$ & 0.33 & 0.00 & 0.00 & 0.00 & 0.00 & 0.00 & 0.00 & 0.00 \\
\hline Sum & 99.25 & 99.21 & 98.24 & 99.17 & 99.39 & 98.74 & 99.27 & 99.06 \\
\hline \multicolumn{9}{|c|}{ Structural formulae based and 4 oxygens } \\
\hline $\mathrm{Si}$ & 0.00 & 0.00 & 0.00 & 0.00 & 0.00 & 0.00 & 0.00 & 0.00 \\
\hline $\mathrm{Al}$ & 1.94 & 1.94 & 1.96 & 1.87 & 1.89 & 1.87 & 1.89 & 1.88 \\
\hline $\mathrm{Ti}$ & 0.01 & 0.01 & 0.01 & 0.01 & 0.01 & 0.01 & 0.01 & 0.00 \\
\hline $\mathrm{Cr}$ & 0.01 & 0.01 & 0.01 & 0.06 & 0.05 & 0.09 & 0.05 & 0.07 \\
\hline $\mathrm{Fe}^{3+}$ & 0.05 & 0.05 & 0.04 & 0.07 & 0.05 & 0.13 & 0.05 & 0.05 \\
\hline $\mathrm{Fe}^{2+}$ & 0.66 & 0.65 & 0.65 & 0.76 & 0.77 & 0.79 & 0.77 & 0.78 \\
\hline $\mathrm{Mn}$ & 0.01 & 0.00 & 0.01 & 0.01 & 0.01 & 0.01 & 0.01 & 0.01 \\
\hline $\mathrm{Mg}$ & 0.33 & 0.34 & 0.34 & 0.23 & 0.22 & 0.19 & 0.21 & 0.21 \\
\hline $\mathrm{Ca}$ & 0.00 & 0.00 & 0.00 & 0.00 & 0.00 & 0.01 & 0.01 & 0.00 \\
\hline $\mathrm{Na}$ & 0.01 & 0.01 & 0.01 & 0.01 & 0.01 & 0.01 & 0.01 & 0.01 \\
\hline $\mathrm{K}$ & 0.00 & 0.00 & 0.01 & 0.00 & 0.00 & 0.00 & 0.00 & 0.00 \\
\hline $\mathrm{F}$ & 0.23 & 0.00 & 0.00 & 0.00 & 0.00 & 0.00 & 0.00 & 0.00 \\
\hline $\mathrm{Zn}$ & 0.13 & 0.13 & 0.12 & 0.35 & 0.33 & 0.48 & 0.36 & 0.35 \\
\hline $\mathrm{X}_{\mathrm{Mg}}$ & 0.33 & 0.34 & 0.34 & 0.23 & 0.22 & 0.19 & 0.21 & 0.21 \\
\hline
\end{tabular}

All samples are aluminous-magnesian granulites. 
Table 7

Representative chemical composition of plagioclase and mesoperthite

\begin{tabular}{|c|c|c|c|c|c|c|c|c|}
\hline Samples & $\mathrm{KJ}-3$ & $\mathrm{KJ}-3$ & BJ-137-A & BJ-137-A & JB-05 & X4-050-B & BJ-137-A & $\mathrm{X} 4-050-\mathrm{B}$ \\
\hline Analysis no. & 1 & 3 & 7 & 20 & 249 & 16 & 21 & 23 \\
\hline Remarks & P11(Core) & P11(Core) & P11(Core) & P11(Core) & P11(Core) & Pl1(Core) & Kfs/Opx2(Core) & Mp1(Core) \\
\hline $\mathrm{SiO}_{2}$ & 59.44 & 59.38 & 58.67 & 58.92 & 55.95 & 59.05 & 63.61 & 63.92 \\
\hline $\mathrm{TiO}_{2}$ & 0.01 & 0.03 & 0.07 & 0.01 & 0.00 & 0.11 & 0.05 & 0.09 \\
\hline $\mathrm{Al}_{2} \mathrm{O}_{3}$ & 26.01 & 26.11 & 25.81 & 25.43 & 28.29 & 25.61 & 18.24 & 18.48 \\
\hline $\mathrm{Cr}_{2} \mathrm{O}_{3}$ & 0.00 & 0.00 & 0.12 & 0.06 & 0.02 & 0.10 & 0.00 & 0.08 \\
\hline $\mathrm{FeO}$ & 0.02 & 0.07 & 0.02 & 0.12 & 0.14 & 0.04 & 0.01 & 0.06 \\
\hline $\mathrm{MnO}$ & 0.00 & 0.00 & 0.00 & 0.00 & 0.00 & 0.12 & 0.00 & 0.00 \\
\hline $\mathrm{MgO}$ & 0.00 & 0.00 & 0.00 & 0.00 & 0.00 & 0.00 & 0.03 & 0.01 \\
\hline $\mathrm{CaO}$ & 6.75 & 7.53 & 7.73 & 7.30 & 10.38 & 7.43 & 0.12 & 0.05 \\
\hline $\mathrm{Na}_{2} \mathrm{O}$ & 7.79 & 7.25 & 6.81 & 6.98 & 5.80 & 7.31 & 0.72 & 0.20 \\
\hline $\mathrm{K}_{2} \mathrm{O}$ & 0.22 & 0.33 & 0.49 & 0.64 & 0.09 & 0.42 & 16.23 & 16.31 \\
\hline Sum & 100.24 & 100.70 & 99.72 & 99.46 & 100.67 & 100.99 & 99.01 & 99.20 \\
\hline \multicolumn{9}{|c|}{ Structural formulae based and 8 oxygens } \\
\hline $\mathrm{Si}$ & 2.66 & 2.63 & 2.63 & 2.66 & 2.49 & 2.63 & 2.98 & 2.98 \\
\hline $\mathrm{Al}$ & 1.32 & 1.36 & 1.38 & 1.35 & 1.48 & 1.34 & 1.01 & 1.02 \\
\hline $\mathrm{Ti}$ & 0.00 & 0.00 & 0.01 & 0.00 & 0.00 & 0.01 & 0.00 & 0.00 \\
\hline $\mathrm{Cr}$ & 0.00 & 0.00 & 0.01 & 0.00 & 0.01 & 0.01 & 0.00 & 0.00 \\
\hline $\mathrm{Fe}^{3+}$ & 0.00 & 0.01 & 0.00 & 0.00 & 0.01 & 0.01 & 0.00 & 0.00 \\
\hline $\mathrm{Fe}^{2+}$ & 0.00 & 0.00 & 0.01 & 0.01 & 0.00 & 0.00 & 0.01 & 0.00 \\
\hline $\mathrm{Mn}$ & 0.00 & 0.00 & 0.00 & 0.00 & 0.00 & 0.01 & 0.00 & 0.00 \\
\hline $\mathrm{Mg}$ & 0.00 & 0.00 & 0.00 & 0.00 & 0.00 & 0.00 & 0.00 & 0.00 \\
\hline $\mathrm{Ca}$ & 0.32 & 0.34 & 0.35 & 0.35 & 0.49 & 0.49 & 0.01 & 0.00 \\
\hline $\mathrm{Na}$ & 0.67 & 0.63 & 0.60 & 0.61 & 0.50 & 0.50 & 0.07 & 0.02 \\
\hline $\mathrm{K}$ & 0.01 & 0.01 & 0.03 & 0.01 & 0.01 & 0.01 & 0.97 & 0.97 \\
\hline $\mathrm{Ab}$ & 66.80 & 62.30 & 61.51 & 62.47 & 50.03 & 62.53 & 6.65 & 1.87 \\
\hline An & 32.00 & 35.80 & 35.60 & 36.09 & 49.46 & 35.09 & 0.63 & 0.25 \\
\hline Or & 1.20 & 1.30 & 2.89 & 1.44 & 1.90 & 2.38 & 92.71 & 97.88 \\
\hline
\end{tabular}

$\mathrm{XH}-050-\mathrm{B}$ is garnet-bearing charnockitic granulite sample and the others are aluminous-magnesian granulites.

et al., 1990) and were formed by the following univariant reaction in the FMAS system (Fig. 3a, b).

$\operatorname{Grt} 1+\operatorname{Sill}( \pm \mathrm{Qtz})=\mathrm{Crd} 1+\operatorname{Spl1}$

We interpret the orthopyroxene and $\mathrm{K}$-feldspar coronae around biotite (Bt1) (Fig. 3f) as the products of the multivariant biotite dehydration-melting reaction in the KFMASH system as suggested by Mohan and Windley (1993):

$\mathrm{Bt} 1+\mathrm{Qtz}=\mathrm{Opx} 2+\mathrm{Kfs}+\mathrm{L}$

The reaction coronae were formed after ductile deformation, since they surround primary biotite (Bt1), which is oriented parallel to the rock foliation. This dehydration reaction leads to the presence of water during metamorphism, and can be used to estimate the value of $\mathrm{aH}_{2} \mathrm{O}$ based on the experimental studies of Hoffer and Grant (1980).

Cordierite porphyroblasts (Crd2) around spinel crystals were formed through the retrograde decompression reaction (Fig. 3c, g), which was studied by Mohan and Windley (1993) and Prakash (1999) in the FMAS system:

$\mathrm{Spl} 2+\mathrm{Qtz}=\mathrm{Crd} 2$

Orthopyroxene (Opx3) plus cordierite (Crd3) symplectite around Grt1 was produced through decompression, and is inferred to record the FMAS divariant reaction 4 (Fig. 3d, e). Similar to symplectites found in the aluminous-granulites from the Central Highland Complex, Sri Lanka (Sajeev and Osanai, 2004), the symplectite orthopyroxene in Bahia granulites also shows an increase in Fe towards the rim (Table 3). The large volume change of this reaction makes it particularly appropriate for geobarometry (Harris and Holland, 1984a,b).

Grt1 + Qtz $=$ Opx3 + Crd3

We suggest that Bt2+Qtz symplectites (Fig. 3h) were formed by back-melting reactions during cooling, as proposed by Kriegsman (2001) in a similar case:

$\mathrm{Opx} 1+\mathrm{Kfs}+\mathrm{L}=\mathrm{Bt} 2+\mathrm{Qtz}$ 
Leucosomes and even orthopyroxene-bearing S-type granites occur in the aluminous-magnesian granulites (Barbosa et al., 2004), showing that melt was formed probably from metapelites along the prograde path and its continued presence was responsible for the formation of biotite (Bt2) during cooling.

\subsection{Garnet-bearing charnockitic granulite}

The studied garnet-bearing charnockitic granulite from HG (Fig. 2) is the product of granulite facies metamorphism of a plutonic protholith. Equilibrium contacts are usually observed between the main mineral phases, although destabilisation of garnet is observed when it is in contact with quartz, which produces orthopyroxene plus plagioclase symplectites. Garnet megacrysts in contact with quartz are armoured by coronae of orthopyroxene (Opx1) and plagioclase (P11), which shows the breakdown of garnet following the silica-saturated FMAS reaction (Raith et al., 1997):

$\mathrm{Grt} 1+\mathrm{Qtz}=\mathrm{Opx} 1+\mathrm{Pl}$

Fine-grained biotite + quartz symplectite was also formed substituting orthopyroxene. These microstructures can be interpreted as products of the multivariant KFMASH reaction:

$\mathrm{Opx}_{1}+\mathrm{Kfs}+\mathrm{L}=\mathrm{Bt} 2+\mathrm{Qtz}$

Barbosa and Sabaté (2004) demonstrated that the rocks of the Jequié block, including $\mathrm{HG}$, were originally in amphibolite facies, and were transformed to granulites during the paleoproterozoic collision. Biotite and hornblende in these granulites are in disequilibrium with orthopyroxene (Barbosa, 1986), showing that melt must have been produced during prograde metamorphism, and may have persisted in small quantities in these rocks, thus being responsible for the formation of retrograde biotite $\mathrm{Bt} 2$ during cooling. On the other hand, retrograde biotite is also found around orthopyroxene and opaque minerals in shear zones formed during the uplift of the Jequie block, by influx of water. This water could also have invaded the garnet-bearing charnockitic granulite, thus forming $\mathrm{Bt} 2$ during cooling.

\section{Thermobarometry}

$P-T$ conditions of the studied rocks were determined using GPT (Reche and Martinez, 1996) and THERMOCALC (Holland and Powell, 1998) softwares (Tables 810). The samples were those described in detail above: aluminous-magnesian granulite samples KJ-3, KJ-1, and BJ-137-A from close to Brejões Dome, and aluminous-magnesian granulite JB-05 and garnetbearing charnockitic granulite $\mathrm{XH}-050-\mathrm{B}$ away from the dome (Fig. 2).

They are rocks with preserved microstructures such as coronae and symplectites that record the decompression processes, yielding information about a number of stages along the retrograde $P-T$ path. On the other hand, little evidence for the prograde $P-T$ path remains. The regression method of Pattison and Bégin (1994) was

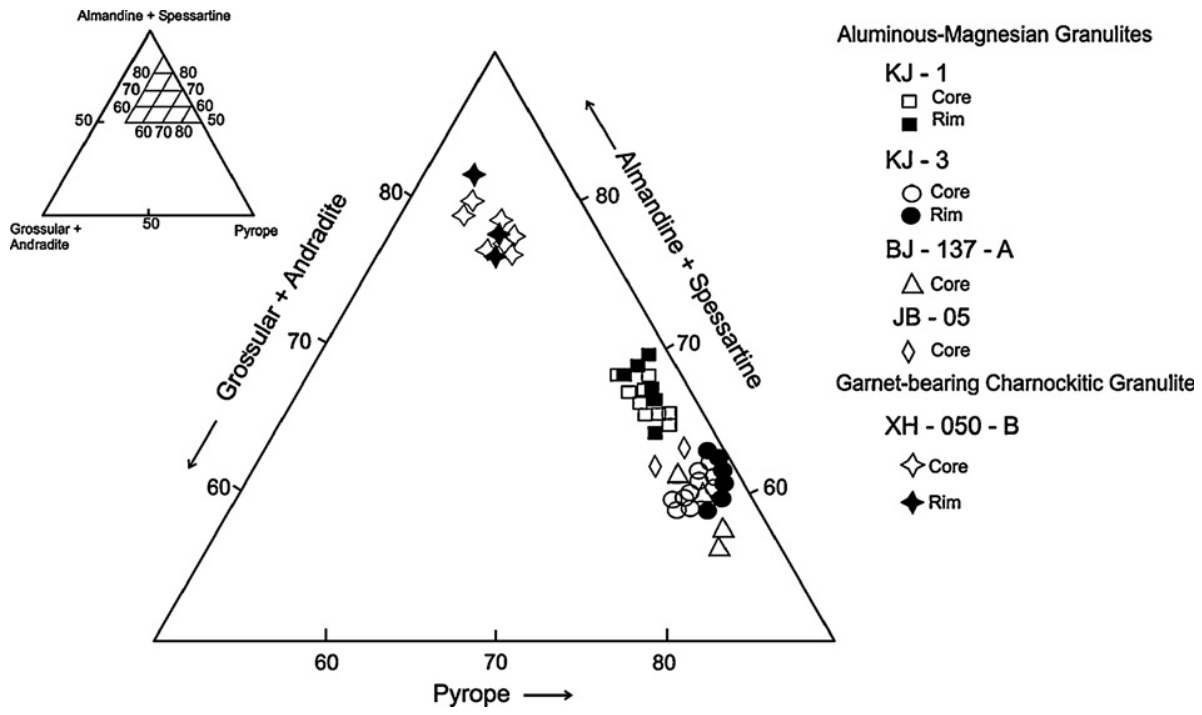

Fig. 4. Garnet compositions of garnet-bearing charnockitic granulite and $\mathrm{Al}-\mathrm{Mg}$ granulite in the pyrope-(grossular + andradite $)-($ almandine + spessartite $)$ diagram. All analysed garnet grains are Grt1. 


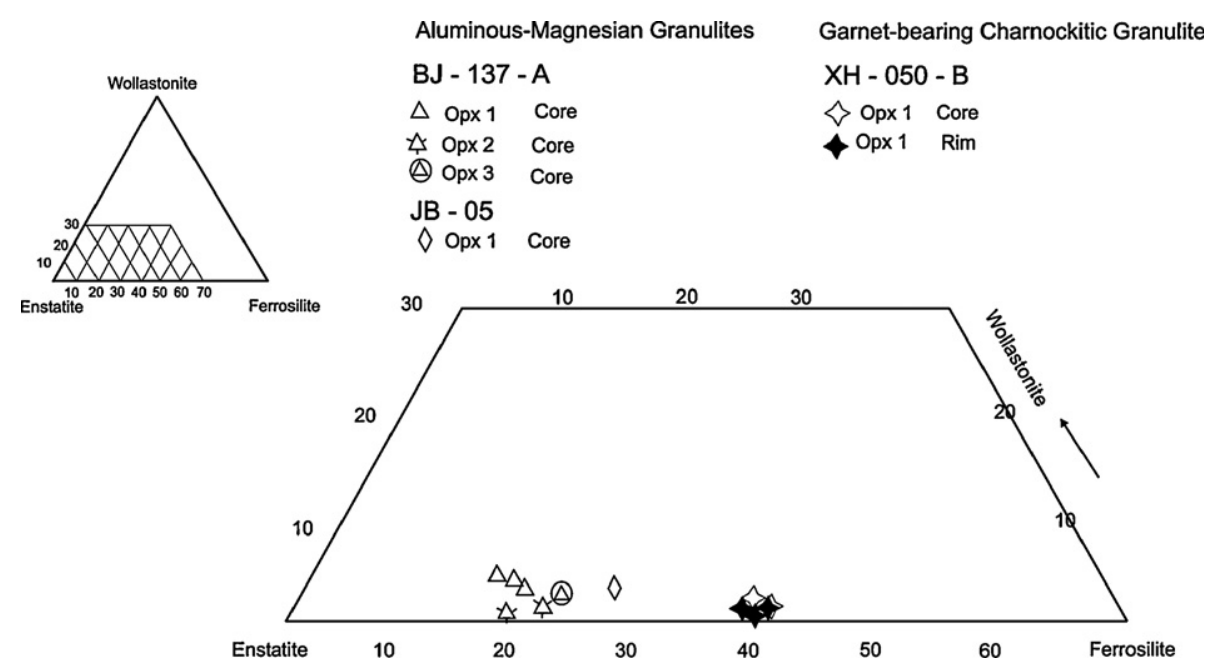

Fig. 5. Orthopyroxene compositions of garnet-bearing charnockitic granulite and aluminous-magnesian granulites in the wollastonite-ferrosiliteenstatite diagram.

applied to retrieve close-to-peak mineral compositions from which close-to-peak $P$ and $T$ estimates were obtained with the Al-orthopyroxene-garnet barometer of Harley and Green (1982), and the garnet-orthopyroxene geothermometer of Harley (1984), respectively, because they are based on the same set of experiments.

Temperatures of $900-1150{ }^{\circ} \mathrm{C}$ were determined using the Grt-Ilm (Pownceby et al., 1991) and Crd-Spl (Vielzeuf, 1983) geothermometers for the $\mathrm{Al}-\mathrm{Mg}$ granulite samples containing spinel (KJ-3, BJ-137-A) close to the Brejões charnockite intrusion (Table 8). The calibration of Holland and Powell (1998) also gives high temperatures $\left(900-1000{ }^{\circ} \mathrm{C}\right)$ for these samples, but indicates lower temperatures $\left(850-870{ }^{\circ} \mathrm{C}\right)$ for the garnet-bearing charnockitic granulite sample (XH-050B) (Table 9) collected farther away from the border. The hercynite + quartz assemblage was formed at a temperature of at least $800^{\circ} \mathrm{C}$ (Ellis et al., 1980; Tobi et al., 1985; Sandiford and Powell, 1986; Waters, 1991). The analysed spinel contains between 0.76 and $2.75 \mathrm{ZnO}$ wt.\%, in the same range as found in spinel from ultrahigh-temperature granulite belts (e.g. Dasgupta et al., 1995; Ouzegane and Boumaza, 1996), while the $\mathrm{Al}_{2} \mathrm{O}_{3}$ content of $6.4 \mathrm{wt} . \%$ in orthopyroxene cores from the aluminous-magnesian granulite (Table 3) also falls within the range of $\mathrm{Al}_{2} \mathrm{O}_{3}$ contents found in orthopyroxene cores from the typical ultrahigh-temperature granulite terrain in Enderby Land, Antarctica (Grew, 1982; Harley et al., 1990).

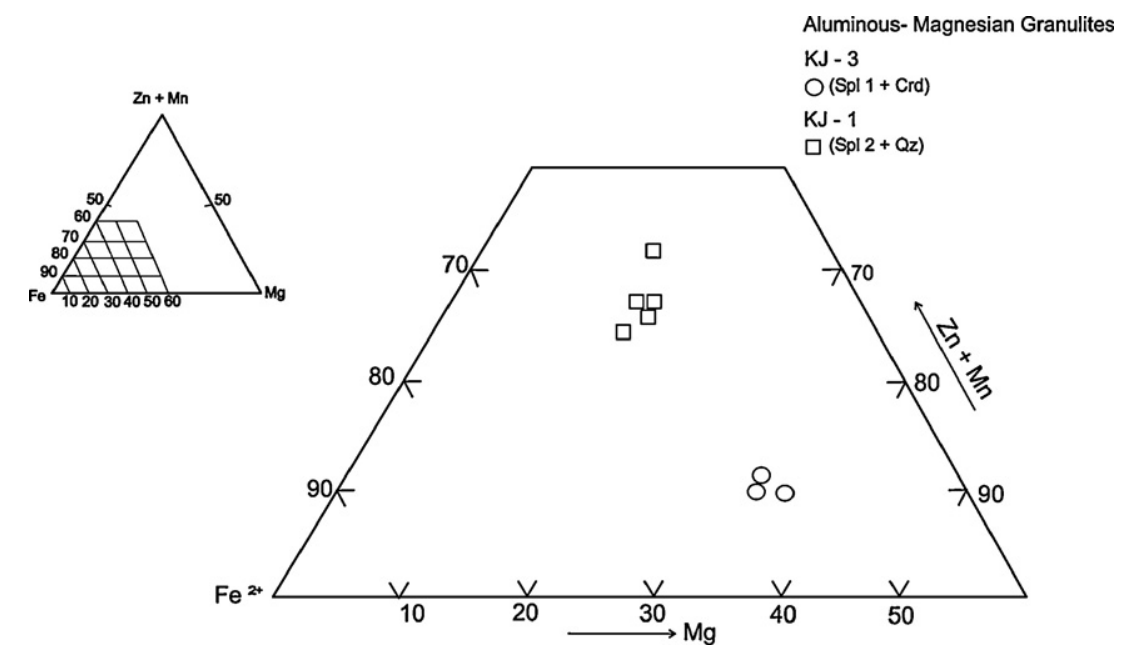

Fig. 6. Spinel composition of hercynite-quartz-bearing granulite in the $\mathrm{Zn}+\mathrm{Mn} / \mathrm{Fe}^{2+} / \mathrm{Mg}$ diagram . 
Table 8

Geothermometry of the studied rocks, according to the method of Reche and Martinez (1996)

\begin{tabular}{|c|c|c|c|c|c|}
\hline \multirow{2}{*}{$\begin{array}{l}\text { Geothermometry/samples } \\
\text { Brejões Dome }\end{array}$} & KJ-3 & KJ-1 & BJ-137 A & JB-05 & XH-050-B \\
\hline & \multicolumn{3}{|c|}{ Samples near Dome } & \multicolumn{2}{|c|}{ Samples far from Dome } \\
\hline \\
\hline \multicolumn{6}{|l|}{ Gt-Crd (7-10 kbars) } \\
\hline Thompson (1976) & $777-836$ & $734-769$ & $721-737$ & & \\
\hline Holdaway and Lee (1977) & $730-790$ & $692-706$ & $695-708$ & & \\
\hline Bhattacharya et al. (1988) & $753-793$ & $737-758$ & $716-731$ & & \\
\hline \multicolumn{6}{|l|}{ Gt-Ilm (7-10 kbars) } \\
\hline Pownceby et al. (1991) & $919-1148$ & $900-1100$ & $991-1090$ & & \\
\hline \multicolumn{6}{|l|}{ Gt-Opx (7-10 kbars) } \\
\hline Sen and Battacharya (1984) & & & $786-809$ & $790-810$ & $657-692$ \\
\hline Harley (1985) & & & $724-741$ & $710-780$ & $617-649$ \\
\hline Lee and Ganguly (1988) & & & $800-819$ & $800-820$ & $697-729$ \\
\hline Lal (1993) & & & $674-696$ & $700-710$ & $690-719$ \\
\hline \multicolumn{6}{|l|}{ Crd-Spl (7-10 kbars) } \\
\hline Vielzeuf (1983) & $818-896$ & $970-902$ & & & \\
\hline
\end{tabular}

Most of calculated $P$ values are between 5 and 8 kbar for all analysed samples, irrespective of the calibration used (Grt-Pl-Bt-Qtz; Grt-Pl-Sil-Qtz; Grt-Rt-SilIlm-Pl-Qtz) (Table 10). Pressure estimates following Holland and Powell (1998) are closer to $8 \mathrm{kbar}$ for both the aluminous-magnesian granulites (KJ-1), and the garnetbearing charnockitic granulite (XH-050-B) (Table 9).

Rocks from the Brejões Dome area (re)-crystallised under low to intermediate pressure (5-8 kbar) granulite facies conditions. Temperatures determined in samples collected away from the dome are in the order of $850{ }^{\circ} \mathrm{C}$, similar to that found elsewhere in the southern Bahia highgrade region (Barbosa, 1986, 1990). Samples from close to the dome, however, record higher temperatures of about $900-1000{ }^{\circ} \mathrm{C}$ which are typical of ultrahightemperature granulite conditions (Harley, 1998). This is confirmed by the $P-T$ conditions calculated using the protocol of Pattison and Bégin (1994) for garnetorthopyroxene pairs in aluminous-magnesian granulite (sample BJ-137-A). The calculated $P$ is $12.0 \pm 2.0 \mathrm{kbar}$ at $T$ of $960 \pm 40-60{ }^{\circ} \mathrm{C}$. The errors are after Harley (1984) who assumes \pm 0.1 as the maximum error in the calculated $K_{\mathrm{D}}$. The data suggest that the Brejões charnockite intrusion was an additional heat source which modified the regional granulite facies metamorphism.

\section{Textural interpretation and $\boldsymbol{P}-\boldsymbol{T}$ history}

The high-temperature assemblages ( $\mathrm{Spl}+\mathrm{Qtz})$ and $(\mathrm{Opx} 1+\mathrm{Bt} 1+\mathrm{Grt} 1+\mathrm{Sill}+\mathrm{Qtz})$ were formed, and the reaction $\mathrm{Grt} 1+$ Sill $( \pm \mathrm{Qtz})=\mathrm{Crd} 1+\mathrm{Spl1}$ occurred at about 7-8 kbar and $1100{ }^{\circ} \mathrm{C}$ and can be explained by using the FMAS system. The retrograde reactions Spl2+
$\mathrm{Qtz}=\mathrm{Crd} 2$ and $\mathrm{Grt} 1+\mathrm{Qtz}=\mathrm{Opx} 3+\mathrm{Crd} 3$, which mainly occurred during orogenic decompression and cooling, can also be explained by using the FMAS system according to Harris and Holland (1984a,b), Harley and Hensen (1990) and Waters (1991). For reaction $\mathrm{Spl}+\mathrm{Qtz}=\mathrm{Crd} 2$ at high temperature we used $\mathrm{X}_{\mathrm{Mg}}=0,7$. The partial pressure of oxygen $\left(f \mathrm{O}_{2}\right)$ is a major factor controlling the stability of the assemblages and reactions (Hensen, 1986). The mineral reactions and the presence of ilmenite inclusions in garnet are consistent with $f \mathrm{O}_{2}$ remaining low throughout their evolution.

On the other hand, reactions $\mathrm{Bt} 1+\mathrm{Qtz}=\mathrm{Opx} 2+\mathrm{Kfs}+\mathrm{L}$ and $\mathrm{Opx} 2+\mathrm{Kfs}+\mathrm{L}=\mathrm{Bt} 1+\mathrm{Qtz}$ can be explained by using the KFMASH system as suggested by Mohan and Windley (1993). The first multivariant dehydration

Table 9

Geothermobarometry of the studied rocks according to the method of Holland and Powell (1998)

\begin{tabular}{|c|c|c|c|c|c|c|}
\hline $\begin{array}{l}\text { Geobarometry/ } \\
\text { samples }\end{array}$ & $\begin{array}{l}\mathrm{KJ}- \\
3\end{array}$ & KJ-1 & $\begin{array}{l}\text { BJ- } \\
137-A\end{array}$ & $\begin{array}{l}\text { BJ- } \\
137-A\end{array}$ & $\begin{array}{l}\text { JB- } \\
05\end{array}$ & $\begin{array}{l}\text { XH- } \\
050-\mathrm{B}\end{array}$ \\
\hline $\begin{array}{l}\text { Dome de } \\
\text { Brejoes }\end{array}$ & \multicolumn{2}{|c|}{$\begin{array}{l}\text { Samples } \\
\text { near Dome }\end{array}$} & & & \multicolumn{2}{|c|}{$\begin{array}{l}\text { Samples far } \\
\text { from Dome }\end{array}$} \\
\hline \multicolumn{7}{|c|}{$\begin{array}{l}\text { Holland and Powell (1998) } \\
\text { (Pl-Opx-Gt- } \\
\text { Bt-Ilm-Fk) }\end{array}$} \\
\hline & & $\begin{array}{l}1051{ }^{\circ} \mathrm{C} ; \\
7.5 \mathrm{~Kb} \\
984{ }^{\circ} \mathrm{C} \\
8.2 \mathrm{~Kb} \\
985{ }^{\circ} \mathrm{C} \\
8.2 \mathrm{~Kb} \\
900{ }^{\circ} \mathrm{C} \\
7.3 \mathrm{~Kb}\end{array}$ & & & & $\begin{array}{l}887^{\circ} \mathrm{C} ; \\
7.1 \mathrm{~Kb} \\
889^{\circ} \mathrm{C} ; \\
7.3 \mathrm{~Kb} \\
850^{\circ} \mathrm{C} ; \\
7.3 \mathrm{~Kb}\end{array}$ \\
\hline
\end{tabular}


Table 10

Geobarometry of the studied rocks, according to the method of Reche and Martinez (1996)

\begin{tabular}{|c|c|c|c|c|c|c|}
\hline Geobarometry/samples & KJ-3 & KJ-1 & \multirow[t]{2}{*}{ BJ-137-A } & \multirow[t]{2}{*}{ BJ-137-A } & JB-05 & XH-050-B \\
\hline Brejões Dome & \multicolumn{2}{|c|}{ Samples near Dome } & & & \multicolumn{2}{|c|}{ Samples far from Dome } \\
\hline \multicolumn{7}{|l|}{ Reche and Martinez (1996) } \\
\hline Gt-Pl-Bt-Qz $\left(850-1100^{\circ} \mathrm{C}\right)$ & & & & $\left(700-850^{\circ} \mathrm{C}\right)$ & & \\
\hline Hoisch (1990) (Mg) & & & $7.6-9.0$ & $5.4-6.1$ & $4.6-5.9$ & $6.7-8.2$ \\
\hline Hoisch (1990) (Fe) & & & $7.4-9.5$ & $6.1-7.0$ & $3.9-5.0$ & $9.1-11.6$ \\
\hline Gt-Pl-Sill-Qz $\left(850-1100{ }^{\circ} \mathrm{C}\right)$ & & & & $\left(700-850^{\circ} \mathrm{C}\right)$ & & \\
\hline Newton and Haselton (1981) & $7.6-9.9$ & $6.8-9.1$ & $7.1-9.3$ & $6.0-6.8$ & $5.4-6.9$ & \\
\hline Hodges and Spear (1982) & $5.7-8.1$ & $5.1-7.1$ & $5.3-7.5$ & $4.4-5.0$ & & \\
\hline Ganguly and Saxena (1984) & $7.4-8.9$ & $6.7-8.9$ & $7.2-9.6$ & $5.4-6.6$ & $5.0-6.5$ & \\
\hline Hodges and Crowley (1985) & $7.4-9.3$ & $6.7-8.6$ & $7.0-8.8$ & $5.5-6.7$ & $5.0-6.4$ & \\
\hline Koziol (1989) & $9.2-11.7$ & $8.5-10.9$ & $8.6-11.1$ & & $7.0-9.0$ & \\
\hline Koziol and Newton (1989) & $8.3-10.6$ & $7.6-9.7$ & $7.9-10.0$ & $6.2-7.6$ & $6.0-7.7$ & \\
\hline Gt-RT-Sil-Ilm-Pl-Qz & & & & $\left(700-850^{\circ} \mathrm{C}\right)$ & & \\
\hline Bohlen et al. (1983) & $6.8-7.6$ & $6.8-7.6$ & $8.7-10.0$ & $4.7-6.8$ & & \\
\hline Bohlen et al. (1983) & $4.8-6.1$ & $5.1-4.8$ & $6.7-7.9$ & $6.2-7.0$ & & \\
\hline Bohlen and Liotta (1986) & $7.3-9.8$ & $6.9-8.3$ & $6.8-8.2$ & $4.1-5.2$ & & \\
\hline
\end{tabular}

reaction, valid for $P_{\mathrm{H} 2 \mathrm{O}}=P_{\text {Total }}$, moves to a higher temperature, probably due to a reduced water activity that could be caused by an influx of $\mathrm{CO}_{2}$ during granulitization (Newton, 1992). Corroded blebs of biotite within orthopyroxene suggest that this reaction did occur. Textural evidence for the second hydratation in the form of biotite plus quartz symplectites around orthopyroxene shows that the first reaction was reversed to produce the second reaction.

\section{Tectonic setting and conclusions}

Low to intermediate pressure and high temperature conditions usually prevailed in Archean/Paleoproterozoic high-grade metamorphic terrains. Many granulite terrains, especially those with hercynite + quartz-bearing granulite, cooled from very hot transient conditions. The most generally applicable models involve a combination of magmatic advection and lithospheric thinning (Waters,

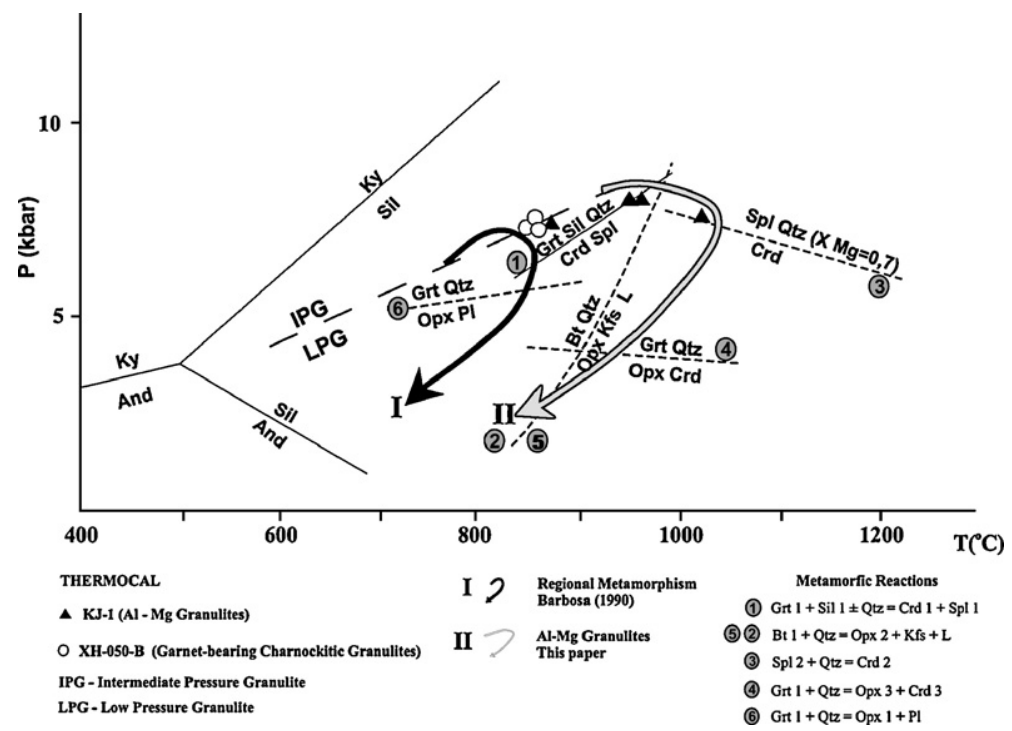

Fig. 7. $P-T$ path of the Brejões Dome area. The higher $T$ path was obtained with the samples near the Brejões Dome and the lower $T$ path (Barbosa, 1990 ) is from the rocks away from the dome. Reactions Grt1 + Sill \pm Qtz $=$ Crd1 + Spl1, Sp12 + Qtz $=$ Crd2 and Grt1 +Qtz $=$ Opx3 + Crd3, observed in rocks of this study, are from Harris and Holland (1984a,b), Harley and Hensen (1990), Waters (1991) and Mohan and Windley (1993). The reactions are calculated using the thermodynamic data base of Holland and Powell (1998). IPG-intermediate pressure granulite and LPG-low pressure granulite (Green and Ringwood, 1972). Aluminium silicates triple point is from Holdaway (1971). 
1991). Thermal aureoles around magmatic intrusions contain high-grade granulite with hercynite + quartz paragenesis (Berg, 1977). The hercynite + quartz association, found in some granulite xenoliths in basaltic rocks, also indicates metamorphic temperatures in excess of $900{ }^{\circ} \mathrm{C}$ (Vielzeuf, 1983).

Clockwise $P-T$ paths are characteristic of geotectonic regions dominated by collision/obduction. Thickening of the continental crust is followed by rapid uplift and erosion, in general at plate margins (England and Richardson, 1977).

The metamorphic path of the granulite region of southern Bahia (Fig. 2) is clockwise, with low/ intermediate pressures $(5-8 \mathrm{~Kb})$ at high temperature $\left(850-870{ }^{\circ} \mathrm{C}\right)$ (Barbosa, 1990) (Fig. 7). It is admitted that this metamorphism was the consequence of crustal thickening, due to the collision with Archean segments (Fig. 8a, b, c), followed by rapid uplift and erosion
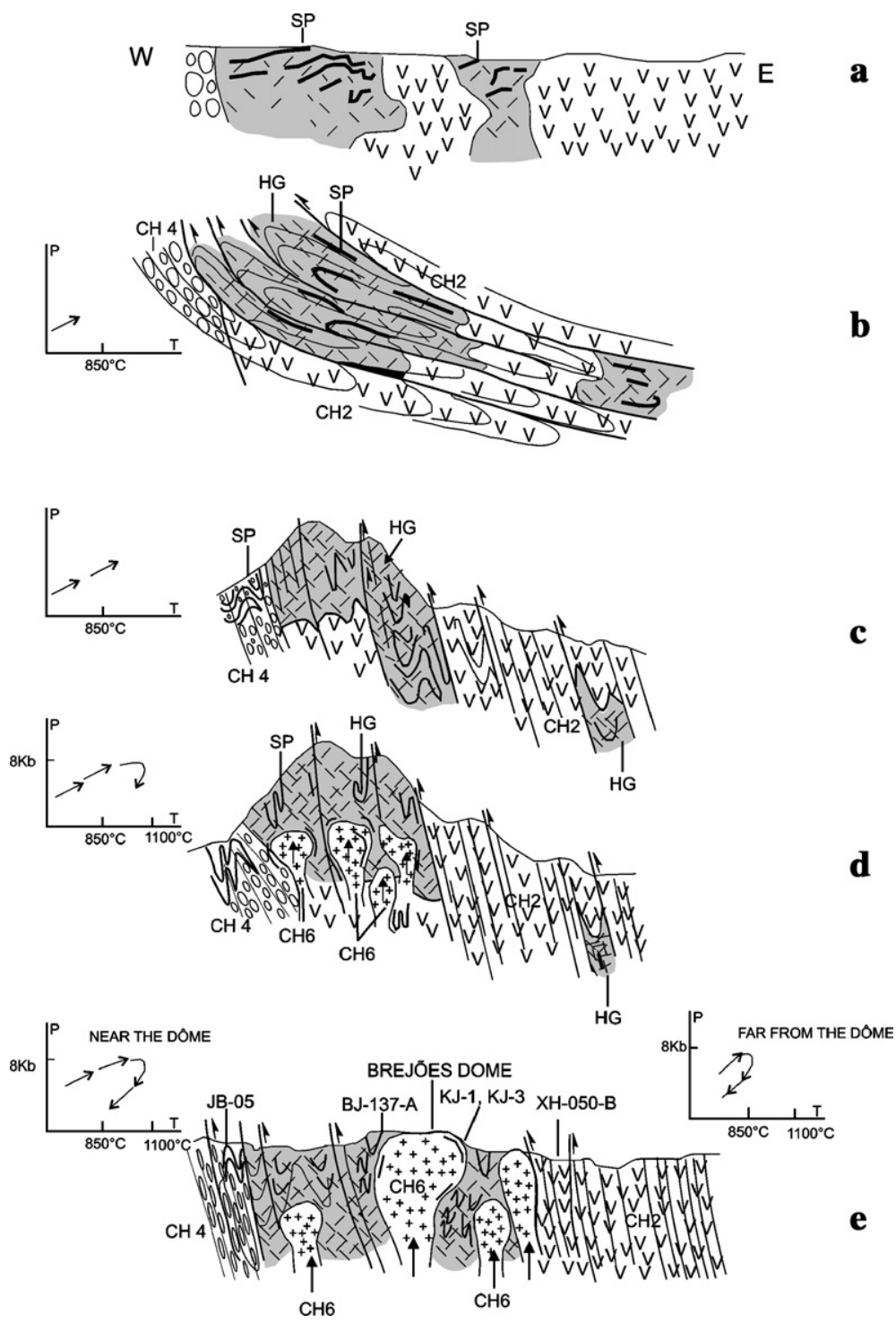

Fig. 8. Geotectonic evolution and $P-T$ paths of the Brejões Dome area. a-Intrusion of enderbitic-charnockitic granulites protoliths, dated at 2.7$2.8 \mathrm{Ga}$, within the Archean crust with supracrustal rocks (SP). b and c - Paleoproterozoic crustal thickening resulting in granulite facies metamorphism ( $\cong 2.04 \mathrm{Ga}$ ) and deformation: first phase of sub-horizontal tectonics (F1), resulting in large west vergent recumbent folds (e.g. Irajuba nappe, in CH4, Fig. 3); second coaxial phase (F2); third phase (F3), represented by vertical shear zones, sub-parallel to (F2) axial planes. d - Late charnockite intrusions (CH6), such as the Brejões and Santa Inês domes, leading to an increase of thermal gradient around 2.04 Ga. e - Present situation of the Brejões Dome area and sampling localities close to the Brejões Dome (low/intermediate pressure and ultrahigh-temperature metamorphism) and away from the dome (low/intermediate pressure and high temperature). 
(Barbosa, 1990; Figueiredo and Barbosa, 1993). The present study corroborates the clockwise $P-T$ path already recorded for the rocks of the high-grade region of southern Bahia. Textural relationships record reactions such as $\mathrm{Grt1}+\mathrm{Sil} \pm \mathrm{Qtz} \Rightarrow \mathrm{Crd1}+\mathrm{Spl1}$ (Reaction 1), and $\mathrm{Bt} 1+\mathrm{Qtz}=\mathrm{Opx} 2+\mathrm{Kfs}+\mathrm{L}$ (Reaction 2), which indicate temperature increase (and/or low $\left.\mathrm{aH}_{2} \mathrm{O}\right)$. These reactions were followed by reactions $\mathrm{Spl} 1+\mathrm{Qtz}=\mathrm{Crd} 2$ (Reaction 3), Grt1 +Qtz=Opx3 $+\mathrm{Crd} 3$ (Reaction 4), $\mathrm{Opx} 1+\mathrm{Kfs}+\mathrm{L}=\mathrm{Bt} 2+\mathrm{Qtz}$ (Reaction 5) and Grt1 +Kfs + $\mathrm{L}=\mathrm{Bt} 2+\mathrm{Qtz}$, which record reduction of $P$ and $T$ (Fig. 7). On the other hand, samples from close to the contact of the Brejões Dome also define a clockwise path at a higher temperature than that recorded in samples away from the dome. It is suggested that the intrusion of the CH6 Brejões charnockite diapir, generated by underplating of the lower crust by magmas which provoked its partial melting, was responsible for the local temperature rise above the peak regional metamorphic temperature (Fig. 8d, e). The hercynite-quartz assemblage in supracrustal rocks surrounding the Brejões dome suggests a very high temperature $\left(>1000{ }^{\circ} \mathrm{C}\right)$ for the $\mathrm{CH} 6$ magmas which would have induced partial melting in adjacent supracrustal rocks, forming garnet-cordierite-bearing granitic melts. Finally, it is uncommon to find evidence for the reaction $\mathrm{Bt} 1+\mathrm{Qtz}=\mathrm{Opx} 2+\mathrm{Kfs}+\mathrm{L}$ occurring in both directions, and we believe that this was possible here because the instrusion of the CH6 Brejões charnockite diapir raised the temperature in the area.

\section{Acknowledgements}

We acknowledge a fellowship from Project 381/02CAPES-COFECUB, the financial support from CBPMCompanhia Baiana de Pesquisa Mineral and CNPqConselho Nacional de Desenvolvimento Científico e Tecnológico, Brazil.

\section{References}

Alibert, C., Barbosa, J.S.F., 1992. Âges U-Pb déterminés à la "SHRIMP" sur des zircons du Complex de Jequié, Craton du São Francisco, Bahia, Brésil. 14 Réun. Sci. Terre (RST), Toulouse, France, p. 4.

Almeida, F.F.M., 1977. O Craton do São Francisco. Rev. Bras. Geociênc. 7, 349-364.

Almeida, F.F.M., Hasui, Y., Brito Neves, B.B., 1976. The upper precambrian of South America. Boletim do Instituto de Geociências, vol. 7. University of São Paulo, Brazil, pp. 45-80.

Barbosa, J.S.F., 1986. Constitution lithologique et métamorphique de la région granulitique du sud de Bahia, Brésil. PhD thesis. Université Paris VI. França. 300 pp.

Barbosa, J.S.F., 1990. The granulites of the Jequié Complex and Atlantic Mobile Belt, Southern Bahia, Brazil—an expression of Achaean
Proterozoic Plate Convergence. In: Vielzeuf, D., Vidal, P. (Eds.), Granulites and Crustal Evolution. Clermont Ferrand (France). NATO ASI Series, vol. C311. Springer-Verlag Proc. ARW, pp. 195-221.

Barbosa, J.S.F., Dominguez, J.M.L., 1996. Texto Explicativo para o Mapa Geológico ao Milionésimo. SICM/SGM, Salvador. (Special Edition), $400 \mathrm{pp}$.

Barbosa, J.S.F., Fonteilles, M., 1991. Síntese sobre o metamorfismo da região granulítica do sul da Bahia, Brasil. Rev. Bras. Geociênc. 21 (4), 328-341.

Barbosa, J.S.F., Sabaté, P., 2002. Geological features and the Paleoproterozoic collision of four Archaean crustal segments of the São Francisco Craton, Bahia, Brazil. A synthesis. An. Acad. Bras. Ciênc. 74 (2), 343-359.

Barbosa, J.S.F., Sabaté, P., 2004. Archean and paleoproterozoic crust of the São Francisco Cráton, Bahia, Brazil: geodynamic features. Precambrian Res. 133, 1-27.

Barbosa, J.S.F., Martin, H., Peucat, J.J., 2004. Archean/paleoproterozoic crustal domic evolution of the Lage, Mutuipe, Brejões and Santa Ines Région. Jequié Block, Bahia, Brazil. Precambrian Res. 135, 105-131.

Berg, J.H., 1977. Dry granulite mineral assemblages in the contact aureoles of the Nain complex, Labrador. Contrib. Mineral. Petrol. $64,33-52$.

Bhattacharya, A., Mazundar, A.C., Sen, S.K., 1988. Fe-Mg mixing in cordierite; constraints from natural data and implications for cordierite-garnet geothermometry in granulites. Am. Mineral. 73 (3-4), 338-344.

Bohlen, S.R., Liotta, J.J., 1986. Barometer for garnet amphibolites and garnet granulites. J. Petrol. 27 (5), 1025-1034.

Bohlen, S.R., Wall, V.J., Boettcher, A.L., 1983. Geobarometry in granulites. In: Saxena, S.K. (Ed.), Kinetics and Equilibrium in Mineral Reactions. Springer Verlag, NY, United States, pp. 141-147.

Clarke, G.L., Powel, R., Guiraud, M., 1989. Low-pressure granulite facies metapelitic assemblages and corona textures from Mac Robertson Land east Antarctica: the importance of $\mathrm{Fe}_{2} \mathrm{O}_{3}$ and $\mathrm{TiO}_{2}$ in accounting for spinel-bearing assemblages. J. Metamorph. Geol. 7, 323-335.

Dasgupta, S., Sengupta, P., Ehl, J., Raith, M., Bardhan, S., 1995. Reaction textures in a suite of spinel granulites from the eastern Ghats Belts, India: evidence for polymetamorphism, a partial petrogenetic grid in the system KFMASH and the roles of $\mathrm{ZnO}$ and $\mathrm{Fe}_{2} \mathrm{O}_{3}$. J. Petrol. 36 (2), 435-461.

Ellis, D.J., Sheraton, J.W., England, R.N., Dallwitz, W.B., 1980. Osumulite-sapphirine-quartz granulites from Enderby Land, Antarctica-mineral assemblages and reactions. Contrib. Mineral. Petrol. 72, 353-367.

England, P.C., Richardson, S.W., 1977. The influence of erosion upon the mineral facies rocks from different metamorphic environments. J. Geol. Soc. (Lond.) 134, 201-213.

Figueiredo, M.C.H., Barbosa, J.S.F., 1993. Terrenos Metamórficos de Alto Grau do Craton do São Francisco. In: Dominguez, J.M.L., Misi, A. (Eds.), O Craton do São Francisco. Salvador, SBG/SGM/ CNPq (Special Publication), pp. 63-83.

Ganguly, J., Saxena, S.K., 1984. Mixing properties of aluminosilicate garnets: constraints from natural and experimental data and applications to geothermo-barometry. Am. Mineral. 69, 88-97.

Green, D.H., Ringwood, A.E., 1972. A comparison of recent experimental data on the gabbro-garnet granulite-eclogite transition. J. Geol. 80, 277-288.

Grew, H., 1982. Osumilite in the sapphirine-quartz terrane of Enderby Land, Antartica : implications for osumilite petrogenesis in the granulite facies. Am. Mineral. 67, 762-787. 
Gonçalves, P., Nicollet, C., Montel, J.-M., 2004. Petrology and in situ $\mathrm{U}-\mathrm{Th}-\mathrm{Pb}$ Monazite Geochronology of ultrahigh-temperature metamorphism from the Andriamena Mafic Unit, North-Central Madagascar. Significance of a petrographical $P-T$ path in a polymetamorphic context. J. Petrol. 45 (10), 1923-1957.

Harley, S.L., 1984. The solubility of alumina in orthopyroxene coexisting with garnet in $\mathrm{FeO}-\mathrm{MgO}-\mathrm{Al}_{2} \mathrm{O}_{3}-\mathrm{SiO}_{2}$ and $\mathrm{CaO}-$ $\mathrm{FeO}-\mathrm{MgO}-\mathrm{Al}_{2} \mathrm{O}_{3}-\mathrm{SiO}_{2}$. J. Petrol. 25 (3), 665-696.

Harley, S.L., 1985. Garnet-orthopyroxene-bearing granulites from Enderby Land, Antarctica: metamorphic pressure temperature time evolution of the Archaean Napier Complex. J. Petrol. 26, 819-856.

Harley, S.L., 1998. An appraisal of peak temperatures and termal historiesin ultrahigh-temperature (UHT) crustal metamorphism: the significance of aluminous orthopyroxene. Mem. Natl. Inst. Polar Res., Spec. Issue 53, 49-73.

Harley, S.L., Green, D.H., 1982. Garnet-orthopyroxene barometry for granulites and peridotites. Nature 300, 697-701.

Harley, S.L., Hensen, B.J., 1990. Archaean and Proterozoic high-grade terranes of East Antarctica $\left(40-80^{\circ} \mathrm{E}\right)$ : a case study of diversity in granulite facies metamorphism. In: Ashworth, J.R., Brown, M. (Eds.), High Temperature Metamorphism and Crustal Anatexis. Unwin Himan, London, pp. 320-370.

Harley, S.L., Hensen, B.J., Sheraton, J.W., 1990. Two-stage decompression in orthopyroxene-sillimanite granulites from Forefinger Point, Enderby Land, Antarctica: implications for the evolution of the Archean Napier Complex. J. Metamorph. Geol. 8, 591-613.

Harris, N.B.W., Holland, T.J.B., 1984a. The significance of cordieritehypersthene assemblages from the Beitbridge of the Central Limpopo Belt; evidence for rapid decompression in the Archaean? Am. Mineral. 69, 1036-1049.

Harris, N.B.W., Holland, T.J.B., 1984b. The significance of cordierite-hypersthene assemblages from the Beitbridge region of the Central Limpopo Belt; evidence for rapid decompression in the Archaean? Am. Mineral. 69, 1036-1049.

Hensen, B.J., 1986. Theoretical phase relations involving cordierite and garnet revisited: the influence of oxygen fugacity on the stability of sapphirine and spinel in the system $\mathrm{Mg}-\mathrm{Fe}-\mathrm{Al}-\mathrm{Si}-\mathrm{O}$. Contrib. Mineral. Petrol. 92, 362-367.

Hensen, B.J., 1987. $P-T$ grids for silica-undersaturated granulites in the systems MAS $(N+4)$ and FMAS $(N+3)$ tools for the derivation of $P-T$ paths of metamorphism. J. Metamorph. Geol. 5, 255-271.

Hodges, K.V., Crowley, P., 1985. $P-T$ paths from garnet zoning; a new tectonic for deciphering tectonic processes in crystalline terrains: discussion and reply. Geology 13 (1), 80-81.

Hodges, K.V., Spear, F.S., 1982. Geothermometry, geobarometry garnet closure temperatures and the $\mathrm{Al}_{2} \mathrm{SiO}_{5}$ triple point, Mt. Moosilauke, N.H. Trans.-Am. Geophys. Union 62 (45), 1060.

Hoffer, E., Grant, J.A., 1980. Experimental investigation of the formation of cordierite-orthopyroxene parageneses in pelitic rocks. Contrib. Mineral. Petrol. 73, 15-22.

Hoisch, T.D., 1990. Empirical calibration of six geobarometers for the mineral assemblage quartz + muscovite + biotite + plagioclase + garnet. Contrib. Mineral. Petrol. 104 (2), 225-234.

Holdaway, M.J., 1971. Stability of andalusite and the aluminium silicate phase diagram. Am. J. Sci. 271, 97-131.

Holdaway, M.J., Lee, H.Y., 1977. Fe-Mg cordierite stability in high grade pelitic rocks based on experimental, theoretical and natural observations. Contrib. Mineral. Petrol. 63, 175-198.

Holland, T., Powell, R., 1998. An internally consistent thermodynamic data set for phases of petrological interest. J. Metamorph. Geol. 16, 309-343.
Hormann, P.K., Raith, M., Raase, P., Ackermand, D., Seifert, F., 1980. The granulite complex of Finnish Lapland: petrology and metamorphic conditions in the Ivalojoki-Inarijarvi area. Geol. Surv. Finl. 308.

Koziol, A.M., 1989. Recalibration of the garnet-plagioclase$\mathrm{Al}_{2} \mathrm{SiO}_{5}$-quartz (GASP) geobarometre and application to natural parageneses. EOS Trans. Am. Geophys. Union 70 (493 pp.).

Koziol, A.M., Newton, R.C., 1989. Grossular activity-composition relationship in ternary garnets determined by reversed displaced-equilibrium experiments. Contrib. Mineral. Petrol. 103 (4), 423-433.

Kretz, R., 1983. Symbols for rock-forming minerals. Am. Mineral. 68, 277-279.

Kriegsman, L.M., 2001. Partial melting, partial melt extraction and partial back reaction in anatectic migmatites. Lithos 56, 75-96.

Lal, R.K., 1993. Internally consistent recalibrations of mineral equilibria for geothermobarometry involving garnet-orthopyroxene-plagioclase-quartz assemblages and their applications to the South Indian Granulites. J. Metamorph. Geol. 11 (6), 855-866.

Lal, R.K., Ackermand, D., Upadhyay, H., 1987. $P-T-X$ relationships deduced from corona textures in sapphirine-spinel-quartz assemblages from Paderu, southern India. J. Petrol. 28, 1139-1168.

Ledru, P., Cocherie, A., Barbosa, J.S.F., Johan, V., Onstott, T., 1994. Âge du métamorphisme granulitique dans le Craton du São Francisco (Brésil). Implications sur la nature de l'orogène transmazonien. C. R. Acad. Sci. Paris 211, 120-125.

Lee, H.Y., Ganguly, J., 1988. Equilibrium compositions of coexisting garnet and orthopyroxene: experimental determinations in the system $\mathrm{FeO}-\mathrm{MgO}-\mathrm{Al}_{2} \mathrm{O}_{3}-\mathrm{SiO}_{2}$ and applications. J. Petrol. 29, 93-113.

Mohan, A., Windley, B.F., 1993. Crustal trajectory of sapphirinebearing granulites from Ganguvarpatti, South India: evidence for an isothermal decompression path. J. Metamorph. Geol. 11, 867-878.

Moraes, R., Fuck, R.A., 2000. Ultra high temperature metamorphism in Central Brazil: the Barro Alto complex. J. Metamorph. Geol. 18 (4), 345-358.

Moraes, R., Brown, M., Fuck, R.A., Camargo, M.A., Lima, T.M., 2002. Characterization and $P-T$ evolution of melt-bearing ultrahigh-temperature granulite: an example from Anapolis-Ituaçú complex of the Brasilia fold belt, Brazil. J. Petrol. 13, 1673-1705.

Newton, R.C., 1992. An overview of charnockite. Precambrian Res. 55, 399-405.

Newton, R.C., Haselton, H.T., 1981. Thermodinamics of the garnetplagioclase- $\mathrm{AlSiO}_{5}-$ quartz geobarometer. In: Newton, R.C., Navrotsky, A., Wood, B.J. (Eds.), Thermodinamics Minerals and Melts. Springer-Verlag, New York, pp. 131-147.

Nicollet, C., 1990. Crustal evolution of the granulites of Madagascar. In: Vielzeuf, D., Vidal, P. (Eds.), Granulites and Crustal Evolution. NATO ASI Series, vol. C311. Springer-Verlag Proc. ARW, Clermont Ferrand, France, pp. 291-310.

Ouzegane, K., Boumaza, S., 1996. An example of ultrahightemperature metamorphism: orthopyroxene-sillimanite-garnet, saphirine-quartz and spinel-quartz parageneses in $\mathrm{Al}-\mathrm{Mg}$ granulites from In Hihaou, In Ouzzal, Hoggar. J. Metamorph. Geol. 14, 693-708.

Pattison, D.R.M., Bégin, N.J., 1994. Zining patterns in orthopyroxene and garnet in granulites: implications for geothermometry. J. Metamorph. Geol. 12, 387-410.

Pownceby, M.I., Wall, V.J., O’Neill, H.St C., 1991. An experimental study of the effect of $\mathrm{Ca}$ upon garnet-ilmenite $\mathrm{Fe}-\mathrm{Mn}$ exchange equilibria. Am. Mineral. 76 (9-10), 1580-1588. 
Prakash, D., 1999. Cordierite-bearing gneisses from Kodaikanal, South India: textural relationship and $P-T$ conditions. J. Geol. Soc. India 54, 347-358.

Raith, M., Karmakar, S., Brown, M., 1997. Ultra-high-temperature metamorphism and multistage decompressional evolution of sapphirine granulites from the Palni Hill Ranges, southern India. J. Metamorph. Geol. 15, 379-399.

Reche, J., Martinez, F.J., 1996. GPT: an excel spreadsheet for thermobarometric calculations in metapelitic rocks. Comput. Geosci. 22 (7), 775-784.

Sajeev, K., Osanai, Y., 2004. Ultrahigh-temperature metamorphism $\left(1150{ }^{\circ} \mathrm{C}, 12 \mathrm{kbar}\right)$ and multistage evolution of $\mathrm{Mg}-\mathrm{Al}$-rich granulites from the Central Highland Complex, Sri Lanka. J. Petrol. 45 (9), 1821-1844.

Sandiford, M.A., Powell, R., 1986. Deep crustal metamorphism during continental extension: modern and ancient examples. Earth Planet. Sci. Lett. 79, 151-158.

Sen, S.K., Battacharya, A., 1984. An orthopyroxene-garnet thermometer and its applications to the Madras charnockites. Contrib. Mineral. Petrol. 88, 64-71.

Thompson, A.B., 1976. Mineral reactions in pelitic rocks: II. Calculation of some $P-T-X(\mathrm{Fe}-\mathrm{Mg})$ phase relations. Am. J. Sci. 276, 425-454.

Tobi, A.C., Hermans, G.A.E.M., Maijer, C., Jansen, J.B.H., 1985. Metamorphic zoning in the high-grade Proterozoic of RogalandVest Agder, SW Norway. In: Tobi, A.C., Touret, J.L.R. (Eds.), The
Deep Proterozoic Crust in the North Atlantic Provinces. NATO ASI Series, vol. C158. D. Reidel, Dordrecht, pp. 477-497.

Ulm, P., 1993. Program NORM Version 4.0, Calculation of Various Minerals-Norms.

Vielzeuf, D., 1983. The spinel and quartz associations in high-grade xenoliths from Tallante (SE Spain) and their potential use in geothermometry and barometry. Contrib. Mineral. Petrol. 82 (4), 301-311.

Warren, R.G., Hensen, B.J., 1989. The $P-T$ evolution of the Proterozoic Arunta Block, central Australia and its implications for tectonic evolution. In: Daly, J.S., Cliff, R.A., Yardley, B.W.D. (Eds.), Evolution of Metamorphic BeltsSpec. Publ.-Geol. Soc. Lond., vol. 43, pp. 349-355.

Waters, D.J., 1989. Metamorphic evidence for the heating and cooling path of Namaqualand granulites. In: Daly, J.S., Cliff, R.A., Yardley, B.W.D. (Eds.), Evolution of Metamorphic BeltsSpec. Publ.-Geol. Soc. Lond., vol. 43, pp. 357-363.

Waters, D.J., 1991. Hercynite-quartz granulites: phase relations, and implications for crustal processes. Eur. J. Mineral. 3, 367-386.

Wilson, N., 1987. Combined $\mathrm{Sm}-\mathrm{Nd}, \mathrm{Pb} / \mathrm{Pb}$ and $\mathrm{RbSr}$ Geochronology and Isotope Geochemistry in Polymetamorphic Precambrian Terrains: Examples from Brazil and Channel Island, U.K. Master of Science. Oxford University, UK. 101 pp. 